\title{
Stable Throughput and Delay Analysis of a Random Access Network With Queue-Aware Transmission
}

Ioannis Dimitriou and Nikolaos Pappas

The self-archived postprint version of this journal article is available at Linköping University Institutional Repository (DiVA):

http:/ / urn.kb.se/ resolve?urn=urn:nbn:se:liu:diva- 145407

N.B.: When citing this work, cite the original publication.

Dimitriou, I., Pappas, N., (2018), Stable Throughput and Delay Analysis of a Random Access Network With Queue-Aware Transmission, IEEE Transactions on Wireless Communications, 17(5), 3170-3184. https:// doi.org/ 10.1109/TWC.2018.2808195

Original publication available at:

https:/ / doi.org/ 10.1109/TWC.2018.2808195

Copyright: Institute of Electrical and Electronics Engineers (IEEE)

http:// www.ieee.org/ index.html

(C) 2018 IEEE. Personal use of this material is permitted. However, permission to reprint/ republish this material for advertising or promotional purposes or for creating new collective works for resale or redistribution to servers or lists, or to reuse any copyrighted component of this work in other works must be obtained from the IEEE. 


\title{
Stable Throughput and Delay Analysis of a Random Access Network With Queue-Aware Transmission
}

\author{
Ioannis Dimitriou, Nikolaos Pappas Member, IEEE
}

\begin{abstract}
In this work we consider a two-user and a threeuser slotted ALOHA network with multi-packet reception (MPR) capabilities and a queue-aware transmission control. In this setting, the nodes can adapt their transmission probabilities and their transmission parameters based on the status of the other nodes. Each user has external bursty arrivals that are stored in their infinite capacity queues. We focus on the fundamental problem of characterizing the stable throughput region, as well as of investigating the queueing delay. For the two- and the three-user cases we obtain the exact stability region, whereas in the former case we also provide the conditions under which the stability region is a convex set. We perform a detailed mathematical analysis to study the queueing delay in the twouser case by formulating two boundary value problems, the solution of which provide the generating function of the joint stationary probability distribution of the queue size at user nodes. Furthermore, for the two-user symmetric case with MPR we obtain a lower and an upper bound for the average delay without the need of solving a boundary value problem. In addition, we provide a closed form expression for the gap between the lower and the upper bound. The bounds as it is seen in the numerical results appear to be tight. Explicit expressions for the average delay are obtained for the symmetrical model with capture effect. We also provide a closed form expression for the optimal transmission probability that minimizes the average delay in the symmetric capture case. Finally, we evaluate numerically the presented theoretical results.
\end{abstract}

Index Terms-Boundary Value Problem, Stable Throughput Region, Delay Analysis, Random Access.

\section{INTRODUCTION}

Since its creation [1], the ALOHA protocol has gained popularity in multiple access communication systems for its simple nature and the fact that it does not require centralized controllers. This simple scheme attempts transmission randomly, independently, distributively, and based on a simple ACK/NACK feedback from the receiver.

Random access recently re-gained interest due to the increase in the number of communicating devices in $5 \mathrm{G}$ networks. More specifically, because of the need of massive uncoordinated access in large networks [2], [3]. Random access and alternatives and their effect on the operation of LTE and LTE-A are presented in [2], [4], [5]. Recently, the effect of random access in physical layer and in other topics

I. Dimitriou is with the Department of Mathematics, University of Patras, Patra, Peloponnese, Greece. (e-mail: idimit@math.upatras.gr).

N. Pappas is with the Department of Science and Technology, Linköping University, Norrköping SE-60174, Sweden. (e-mail: nikolaos.pappas@liu.se).

This work has been partially supported by the EU project DECADE under Grant H2020-MSCA-2014-RISE: 645705, the European Unions Horizon 2020 research and innovation programme and by the European Unions Horizon 2020 research and innovation programme under the Marie Skodowska-Curie grant agreement No. 643002 (ACT5G). This work was supported in part by ELLIIT and CENIIT. has been studied [6], [7], [8], [9] and the research in this area is in progress. Random access remains an active research area where a lot of fundamental questions remain open even for very simple networks [10], [11].

When the traffic in a network is bursty, a relevant performance measure is the stable throughput or stability region. The exact characterization of the stability region is known to be a difficult problem due to the interaction among the queues. In addition to throughput, delay is another important metric. Recently there is a rapid growth on supporting realtime applications thus, there is a need to provide delay-based guarantees [3], [12]. Therefore, the characterization of the delay is of major importance. However, the exact characterization of delay even in small random access networks is rather difficult and remains unexplored in most of the cases.

In this work, we consider a two-user and a three-user slotted ALOHA network with multi-packet reception capabilities. We also employ a queue-aware protocol, which allow the nodes to adapt their transmission probabilities and their transmission parameters based on the status of the other nodes. We analyze the stable throughput region and study the queueing delay by utilizing the theory of boundary value problems.

The results of our work can be applied directly or indirectly to several real-life cases. One scenario can appear in data collection applications where aggregators are collecting data from sensors in different areas. The aggregators (i.e user nodes) are storing the collected data and they are responsible to transmit them to a remote receiver. Another possible application arises from the problem of sampling of random processes. Random processes are sampled then the samples are stored in queues waiting to be transmitted to a remote destination. The goal of the remote destination is to timely reconstruct the processes from the received samples. These scenarios motivate further the contribution of our work.

\section{A. Related Work}

In the literature there is a vast number of papers that are considering the stable throughput and delay in random access and variations of random access schemes.

The derivation of the stability region of random access systems for bursty sources is known to be a difficult problem above three sources. This is because each source transmits and interferes with the others only when its queue is nonempty. Such queues where the service process of one depends on the status of the others are said to be coupled or interacting. Thus, the individual departure rates of the queues cannot be computed separately without knowing the stationary distribution of the joint queue length process [13]. This is the 
reason why the vast majority of previous works has focused on small-sized networks and only bounds or approximations are known for the networks with larger number of sources [14], [13], [15], [16], [17], [7]. In [18], an approximation of the stability region was obtained based on the mean-field theory for network of nodes having identical arrival rates and transmission probabilities were performed. The work in [19] investigates the stable throughput region of a random access network where the transmitters and receivers are distributed by a static Poisson bipolar process.

Delay analysis in random access networks was studied in [17], [20], [21], [22]. More specifically, in [20] a two-user network with collision channel was studied and expressions for the average delay were obtained. A two-user network with MPR capabilities was considered in [17], [7] and expressions for the average delay were obtained under the strong assumption of the absolute symmetry of the model. In [23] the delay performance of slotted ALOHA in a Poisson network was studied. Delay analysis of random access networks based on fluid models can be found in [24], [25]. The works in [26], [27] utilized techniques from statistical mechanics for throughput and delay analysis. In [28] a service-martingale concept that enables the queueing analysis of a bursty source sharing a MAC channel was proposed.

In the following, we present a recent set of papers considering throughput and/or delay characterization of general random access networks. The work in [29] studied the impact of a full duplex relay in terms of throughput and delay in a multi-user network, where the users were assumed to have saturated traffic. The delay of a random access scheme in the Internet of Things concept was studied in [30]. In [31] throughput with delay constraints was studied in a shared access cognitive network. The delay characterization of larger networks was considered in [32], [33]. In [34] the delay and the packet loss rate of a frame asynchronous coded slotted ALOHA system for an uncoordinated multiple access were also studied.

\section{B. Contribution}

Our contribution in this work can be summarized as follows. We consider the case of the two and three-user wireless network with a common destination. The nodes/sources access the medium in a random access manner and time is assumed to be slotted. Each user has external bursty arrivals that are stored in their infinite capacity queues. We consider multi packet reception (MPR) capabilities at the destination node.

The nodes are accessing the wireless channel randomly and they adapt their transmission probabilities based on the status of the queue of the other nodes. More precisely, a node adapts its transmission characteristics based on the status of the other node in order to exploit its idle slots, and to increase the chances of a successful packet transmission. To the best of our knowledge this variation of random access has not been reported in the literature. The contribution of this work has two main parts focused on the stable throughput region, and the detailed analysis of the queueing delay at users nodes.

1) Stable Throughput Region Analysis: The first part is related to the study of stable throughput. More specifically, we obtain the stability conditions for the case of two and three users. Furthermore, we obtain the conditions where the stability region is a convex set. Convexity is an important property since it corresponds to the case where parallel concurrent transmissions are preferable to a time-sharing scheme. Moreover, we would like also to emphasize that the exact stability region for the case of three nodes with MPR even in the simple random access case (without transmission control) is not known in the literature. The only related paper refers to a collision channel model without transmission control [35].

The main difficulty for characterizing the stability region lies on the interaction of the queues. The interaction of the queues arise when the service rate of a queue depends on the state of the other. A tool to bypass this difficulty is the stochastic dominance technique introduced in [13]. However, the three-user network is more elaborated and the stability region cannot be derived that easily. As mentioned earlier, in the literature the three-user scenario has studied only for the collision channel model.

2) Delay Analysis: The second part of the contribution of this work is the delay analysis. Based on a relation among the values of the transmission probabilities we distinguish the analysis in two cases, which are different both in the modeling, and in the technical point of view. In particular, the analysis leads to the formulation of two boundary value problems (e.g., [20], [36], [37], [38], [39], [40], [41]), the solution of which provide the generating function of the stationary joint probability distribution of the queue size for the two-user case with MPR. This is the key element for obtaining expressions for the average delay at each user node. The analysis is rather complicated and novel. Furthermore, for the two-user symmetric case with MPR, we obtain a lower and an upper bound for the average delay without explicitly computing the generating function for the stationary joint queue length distribution. In addition, we provide in terms of closed form expression, the gap between the lower and the upper bound. The bounds as it is also seen in the numerical results appear to be tight. For the model with capture effect, i.e., a subclass of MPR models, we provide the explicit expression for the average delay. We also obtain in closed form, the optimal transmission probability that minimizes the average delay in the symmetric capture case. Concluding, the analytical results in this work, to the best of our knowledge, have not been reported in the literature.

The rest of the paper is organized as follows. In Section II we present the system model by providing the details of the proposed protocol and the underlying physical layer on the channel model. In Section III we provide the stability region for the two-user case. In Section IV we derive the fundamental functional equation and obtain some important preparatory results for the delay analysis. Section V is devoted to the formulation of two boundary value problems, the solution of which provides the generating function of the joint stationary queue length distribution of user nodes. The expected number of packets and the average delay expressions are also obtained. In Section VI, we provide an alternative approach to obtain the stability conditions for the two-user case, and we also obtain the stability region for the three-users case. In Section VII, 
we obtain explicit expressions for the average delay at each user for the symmetrical system. Finally, numerical examples that provide insights in the system performance are given in Section VIII.

\section{SySTEM MODEL}

\section{A. Network Model}

We consider a slotted random access network consisting of $N=2,3$ users communicating with a common receiver. Each user has an infinite capacity buffer, in which stores arriving and backlogged packets. Packets have equal length and the time is divided into slots corresponding to the transmission time of a packet. Denote by $N_{k, n}$ the number of packets in user node $k$ at the beginning of the $n$th slot. Let also $\left\{A_{k, n}\right\}_{n \in \mathbb{N}^{*}}$ to be a sequence of independent and identically distributed random variables where $A_{k, n}$ is the number of packets arriving in user node $k$, in the time interval $(n, n+1)$, with $E\left(A_{k, n}\right)=\lambda_{k}<$ $\infty, k=1,2$.

Quеиe-aware transmission policy: At the beginning of each slot, there is a possibility for the node $k, k=1,2$, to transmit a packet to the receiver. The receiver has MPR capabilities, and thus more than one concurrent transmission can occur without having a collision. Due to the interference, and the complex interdependence among the nodes we consider the following policy: If both nodes are non empty (i.e., they are both active), node $k$, transmits a packet with probability $\alpha_{k}$, $k=1,2$, independently, with $\bar{\alpha}_{k}=1-\alpha_{k}$. If node 1 (resp. 2) is the only non-empty, it transmits a packet with probability $\alpha_{k}^{*}$, with $\bar{\alpha}_{k}^{*}=1-\alpha_{k}^{*} \cdot{ }^{1}$ Note that in our case, a node is aware about the state of its neighbor. ${ }^{2}$

\section{B. Physical Layer Model}

The MPR channel model used in this paper is a generalized form of the packet erasure model. In the wireless environment, a packet can be decoded correctly by the receiver if the received SINR exceeds a certain threshold. More precisely, suppose that we are given a set $T$ of nodes transmitting in the same time slot. Let $P_{r x}(i, j)$ be the signal power received from node $i$ at node $j$ (when $i$ transmits), and let $\operatorname{SINR}(i, j)$ be the SINR received by node $j$, i.e.,

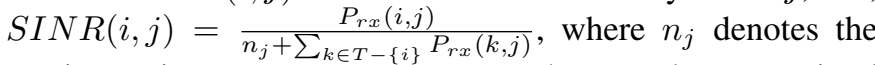
receiver noise power at $j$. We assume that a packet transmitted by $i$ is successfully received by $j$ if and only if $\operatorname{SINR}(i, j) \geq$ $\gamma_{i}$, where $\gamma_{i}$ is the SINR threshold. The wireless channel is subject to fading; let $P_{t x}(i)$ be the transmitting power at node $i$ and $r(i, j)$ be the distance between $i$ and $j$. The power received by $j$ when $i$ transmits is $P_{r x}(i, j)=A(i, j) g(i, j)$ where $A(i, j)$ is a random variable representing channel fading. We assume that the fading model is slow, flat fading, constant during a time slot and independently varying from time slot

\footnotetext{
${ }^{1}$ We consider the general case for $\alpha_{k}^{*}$ instead of assuming directly $\alpha_{k}^{*}=1$. This can handle cases where the node cannot transmit with probability one even if the other node is silent. This scenario for example can occur when the nodes are subject to energy limitations. It is outside of the scope of this work to consider specific reasons when this case can appear but we intent to keep the proposed analysis general.

${ }^{2}$ In a shared access network, it is practical to assume some minimum exchanging information of one bit in this case.
}

to time slot. Under Rayleigh fading, it is known [42] that $A(i, j)$ is exponentially distributed. The received power factor $g(i, j)=P_{t x}(i)(r(i, j))^{-h}$ where $h$ is the path loss exponent with typical values between 2 and 6 . In this study we consider one destination which is common for both nodes, thus $j$ denotes the common destination here and we can also write $\operatorname{SINR}(i, j)=S I N R_{i}$. The success probability of link $i, j$ when the transmitting nodes are in the set $T$ is given by [42]

$$
\begin{aligned}
P_{s}(i, T) & =\operatorname{Pr}\left(S I N R_{i} \geq \gamma_{i}\right) \\
& =\exp \left(\frac{-\gamma_{i} n_{j}}{v(i, j) g(i, j)}\right) \prod_{k \in T-\{i\}}\left(1+\frac{v(k, j) g(k, j)}{v(i, j) g(i, j)}\right)^{-1},
\end{aligned}
$$

where $v(i, j)$ is the parameter of the Rayleigh random variable for fading. According to (1) we denote $P_{i /\{i, j\}}$ to be the success probability of node $i$ when the transmitting nodes are $i$ and $j, i, j=1,2$. More precisely: the strongest user can transmit successfully even in the presence of simultaneous transmissions, if the difference in power is large enough [43] (provided that $S I N R(i, k) \geq \gamma_{k}$ ). If both nodes transmit, but their $S I N R$ are below the threshold $\gamma_{k}$, their transmission is unsuccessful.

Next, we will define for convenience some conditional probabilities on top of the expression given in (1). ${ }^{3}$ We define $P_{1 /\{1,2\}}$ the probability that when both nodes 1 and 2 are transmitting only the transmission from node 1 is successful. Then $P_{1 /\{1,2\}}=\operatorname{Pr}\left(S I N R_{1} \geq \gamma_{1}, S I N R_{2}<\gamma_{2}\right)$. Similarly we can define $P_{2 /\{1,2\}}$. The $P_{1,2 /\{1,2\}}$ is the probability that both packets transmitted by nodes 1 and 2 are transmitted successfully,

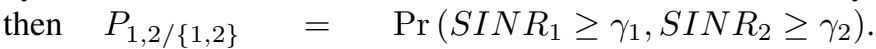
Then we have $P_{s}(1,\{1,2\})=P_{1 /\{1,2\}}+$ $P_{1,2 /\{1,2\}}$. Note that $P_{s}(1,\{1,2\})=$ $\operatorname{Pr}\left(S I N R_{1} \geq \gamma_{1}\right)=\operatorname{Pr}\left(S I N R_{1} \geq \gamma_{1}, S I N R_{2}<\gamma_{2}\right)+$ $\operatorname{Pr}\left(S I N R_{1} \geq \gamma_{1}, S I N R_{2} \geq \gamma_{2}\right)$.

The $P_{0 /\{1,2\}}=\operatorname{Pr}\left(S I N R_{1}<\gamma_{1}, S I N R_{2}<\gamma_{2}\right)$ is the probability where both packets fail to reach the destination when both nodes 1 and 2 are transmitting, then $P_{0 /\{1,2\}}=1-$ $P_{1 /\{1,2\}}-P_{2 /\{1,2\}}-P_{1,2 /\{1,2\}}$. Note that $P_{i /\{i\}}=P_{s}(i,\{i\})$ is the success probability of node $i$ when only $i$-th node transmits but the other one is active (i.e., there are packets stored in its buffer), we denote with $P_{0 /\{i\}}=1-P_{s}(i,\{i\})$ the outage probability respectively. Furthermore, we assume that a node adjusts its transmission parameters such as the transmission power when the other node has an empty queue (i.e is inactive). Thus, the success (resp. outage) probability of node $i$ when the other node is inactive is denoted by $\tilde{P}_{i /\{i\}}$ (resp. $\tilde{P}_{0 /\{i\}}$ ). By allowing this we can consider a simple power control policy where a node can adapt its transmission power when the other node is empty, in order to increase the success probability thus, is reasonable to assume that $\tilde{P}_{i /\{i\}} \geq P_{i /\{i\}}$.

In the case of an unsuccessful transmission the packet has to be re-transmitted in a future timeslot. We assume that the receiver gives an instantaneous error-free feedback (ACK) of all the packets that were successful in a slot at the end of the

\footnotetext{
${ }^{3}$ A similar approach can be found in [17], [7].
} 
TABLE I

BASIC NOTATION

\begin{tabular}{|c|l|}
\hline Symbol & Explanation \\
\hline$N_{k, n}$ & $\begin{array}{l}\text { The number of packets in the user node } k \\
\text { at the beginning of slot } n\end{array}$ \\
\hline$A_{k, n}$ & $\begin{array}{l}\text { The number of packets arriving during }(n, n+1] \\
\text { in user node } k, k=1,2\end{array}$ \\
\hline$D_{k, n}$ & $\begin{array}{l}\text { The number of departures from user node } k, \\
k=1,2, \text { at slot } n\end{array}$ \\
\hline$\lambda_{k}$ & $\begin{array}{l}\text { The expected number of arrivals in user node } k, \\
k=1,2, \text { during a slot }\end{array}$ \\
\hline$a_{k}$ & $\begin{array}{l}\text { Transmission probability of user node } k, \\
k=1,2, \text { when both users are active }\end{array}$ \\
\hline$a_{k}^{*}$ & $\begin{array}{l}\text { Transmission probability of user node } k, \\
k=1,2, \text { when it is the only active node }\end{array}$ \\
\hline$P_{k / T}$ & $\begin{array}{l}\text { Success probability of user node } k, \\
k=1,2, \text { when the transmitting nodes are in } T\end{array}$ \\
\hline$\tilde{P}_{k /\{k\}}$ & $\begin{array}{l}\text { Success probability of user node } k, \\
k=1,2, \text { when it is the only active node }\end{array}$ \\
\hline
\end{tabular}

slot. The nodes remove the successfully transmitted packets from their buffers. The packets that were not successfully transmitted are retained.

Now we can write the expressions for the average service rates $\mu_{1}$ and $\mu_{2}$ seen at node 1 and 2 respectively. The expression for $\mu_{1}$ is given below (similarly we can obtain $\mu_{2}$ ),

$$
\begin{aligned}
& \mu_{1}=\operatorname{Pr}\left(N_{2} \neq 0\right)\left[\alpha_{1} \bar{\alpha}_{2} P_{1 /\{1\}}+\alpha_{1} \alpha_{2}\right. \\
& \left.\times\left(P_{1 /\{1,2\}}+P_{1,2 /\{1,2\}}\right)\right]+\operatorname{Pr}\left(N_{2}=0\right) \alpha_{1}^{*} \tilde{P}_{1 /\{1\}},
\end{aligned}
$$

where $N_{k}=\lim _{n \rightarrow \infty} N_{k, n}, k=1,2$. We can easily see from (2) that the service rate of one queue depends on the status of the other queue. Thus, the queues are coupled. In Section III we bypass this difficulty by applying the stochastic dominance technique to obtain the exact stability region. Regarding the delay analysis we need a different treatment, based on the powerful and technical theory on boundary value problems; see Section V.

Based on the definition in [35], a user's node is said to be stable if $\lim _{n \rightarrow \infty} \operatorname{Pr}\left[N_{k, n}<x\right]=F(x)$ and $\lim _{x \rightarrow \infty} F(x)=$ 1. Loynes' theorem [44] states that if the arrival and service processes of a queue are strictly jointly stationary and the average arrival rate is less than the average service rate, then the queue is stable. If the average arrival rate is greater than the average service rate, then the queue is unstable and the value of $N_{k, n}$ approaches infinity almost surely. The stability region of the system is defined as the set of arrival rate vectors $\boldsymbol{\lambda}=\left(\lambda_{1}, \lambda_{2}\right)$, for which the queues in the system are stable.

\section{STABILITy REgION FOR $N=2$ USERS}

The following theorem provides the stability region for our two-user random access network with queue-aware transmission policy.

Theorem III.1. The stability region $\mathcal{R}$ for a fixed transmission probability vector $\mathbf{p}:=\left[\alpha_{1}, \alpha_{1}^{*}, \alpha_{2}, \alpha_{2}^{*}\right]$ is given by $\mathcal{R}=\mathcal{R}_{1} \cup$
$\mathcal{R}_{2}$ where

$$
\begin{aligned}
& \mathcal{R}_{1}=\left\{\left(\lambda_{1}, \lambda_{2}\right): \lambda_{1}<\alpha_{1}^{*} \tilde{P}_{1 /\{1\}}+\widehat{d}_{1} \frac{\lambda_{2}}{\alpha_{2} \widehat{\alpha}_{1}}, \lambda_{2}<\alpha_{2} \widehat{\alpha}_{1}\right\}, \\
& \mathcal{R}_{2}=\left\{\left(\lambda_{1}, \lambda_{2}\right): \lambda_{2}<\alpha_{2}^{*} \tilde{P}_{2 /\{2\}}+\widehat{d}_{2} \frac{\lambda_{1}}{\alpha_{1} \widehat{\alpha}_{2}}, \lambda_{1}<\alpha_{1} \widehat{\alpha}_{2}\right\},
\end{aligned}
$$

where $\widehat{d}_{k}=d_{k}+\alpha_{1} \alpha_{2} P_{1,2 /\{1,2\}}$ for $k=1,2, d_{1}=$ $\alpha_{1}\left(\bar{\alpha}_{2} P_{1 /\{1\}}+\alpha_{2} P_{1 /\{1,2\}}\right)-\alpha_{1}^{*} \tilde{P}_{1 /\{1\}}, d_{2}=\alpha_{2}\left(\bar{\alpha}_{1} P_{2 /\{2\}}+\right.$ $\left.\alpha_{1} P_{2 /\{1,2\}}\right)-\alpha_{2}^{*} \tilde{P}_{2 /\{2\}}, \widehat{\alpha}_{1}=\bar{\alpha}_{1} P_{2 /\{2\}}+\alpha_{1}\left(P_{2 /\{1,2\}}+\right.$ $\left.P_{1,2 /\{1,2\}}\right), \widehat{\alpha}_{2}=\bar{\alpha}_{2} P_{1 /\{1\}}+\alpha_{2}\left(P_{1 /\{1,2\}}+P_{1,2 /\{1,2\}}\right)$.

Proof. The proof is given in the Appendix A.

Remark 1. Note that $\widehat{d}_{k}$ is the difference of the successful transmission probability of node $k$, when both nodes are active (i.e., both nodes have packets to send) minus the successful transmission probability of node $k$ when the other node is inactive (i.e., only node $k$ has packets to transmit). In the later case due to the lack of interference and since node $k$ senses the other node inactive, it will transmit with a higher probability in order to exploit the idle slot of the other node. In such a case $\tilde{P}_{k /\{k\}} \geq P_{k /\{k\}} \geq P_{k /\{1,2\}} \geq P_{1,2 /\{1,2\}}{ }^{4}$ Thus, from here on we will assume that $\widehat{d}_{k}<0, k=1,2$.

Remark 2. The stability region is a convex polyhedron when the following condition holds $\frac{\alpha_{1} \widehat{\alpha}_{2}}{\alpha_{1}^{*} \hat{P}_{1 /\{1\}}}+\frac{\alpha_{2} \widehat{\alpha}_{2}}{\alpha_{2}^{*} \hat{P}_{2 /\{2\}}} \geq 1$. When equality holds in the previous condition (see also Fig. 1), the region is a triangle and coincides with the case of timesharing. Convexity is an important property since it corresponds to the case when parallel concurrent transmissions are preferable to a time-sharing scheme. Additionally, convexity of the stability region implies that if two rate pairs are stable, then any rate pair lying on the line segment joining those two rate pairs is also stable.

Remark 3. The condition $\frac{\alpha_{1} \widehat{\alpha}_{2}}{\alpha_{1}^{*} \tilde{P}_{1 /\{1\}}}+\frac{\alpha_{2} \widehat{\alpha}_{2}}{\alpha_{2}^{*} \tilde{P}_{2 /\{2\}}} \geq 1$ is the generalized version of the condition that characterizes the $M P R$ capability in the system which was first appeared in [17].

\section{Preparatory Analysis \& Results}

In this section we provide the first part of the analysis that is needed to obtain the expressions for the delay analysis. More explicitly, we derive the fundamental functional equation and we obtain some important results that we will use in the analysis of Section $\mathrm{V}$.

We denote by $Z(x, y)=\lim _{n \rightarrow \infty} E\left(x^{A_{1, n}} y^{A_{2, n}}\right),|x| \leq 1$, $|y| \leq 1$, the moment generating function of the joint arrival process. From here on we will assume geometrically distributed arrival processes (see also [20], [17]) at both stations, i.e., $Z(x, y)=\left[\left(1+\lambda_{1}(1-x)\right)\left(1+\lambda_{2}(1-y)\right)\right]^{-1},|x| \leq$ $1,|y| \leq 1$. We have chosen the geometric distribution for sake of convenience for the delay analysis, and it is not restrictive. The results regarding the stability analysis are also

\footnotetext{
${ }^{4}$ The particular case where $\tilde{P}_{k /\{k\}}=P_{k /\{k\}}=P_{k /\{1,2\}}=P_{1,2 /\{1,2\}}$ is omitted since there is no coupling between the queues and the analysis becomes trivial.
} 


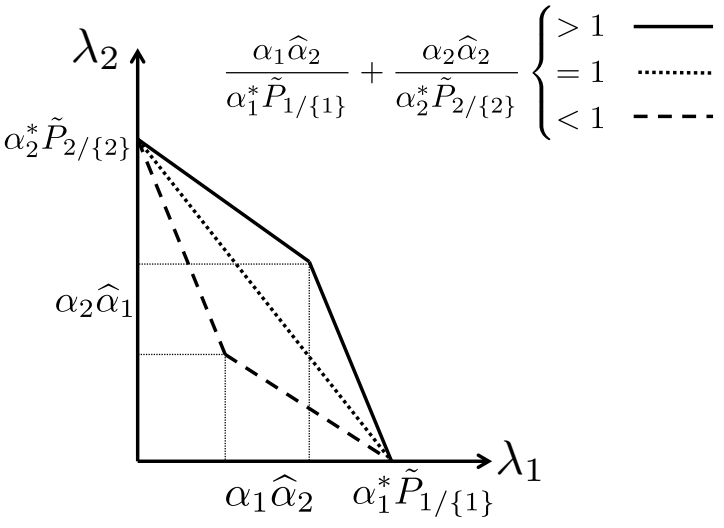

Fig. 1. The Stability Region described in Theorem III.1.

not affected by this assumption. There, $Y_{n}=\left(N_{1, n}, N_{2, n}\right)$ is a two-dimensional discrete time Markov chain with state space $V=\{(i, j): i, j=0,1,2, \ldots\}$. The queues of both users evolve as $N_{k, n+1}=\left[N_{k, n}-D_{k, n}\right]^{+}+A_{k, n}, k=1,2$, where $D_{k, n}$ is the number of departures from user $k$ queue at time slot $n$. Denote by $H(x, y)=\lim _{n \rightarrow \infty} E\left(x^{N_{1, n}} y^{N_{2, n}}\right),|x| \leq$ $1,|y| \leq 1$, the generating function of the stationary joint probability distribution of the number of stored packets at user nodes. Then, the queue evolution equation implies,

$$
\begin{aligned}
& E\left(x^{N_{1, n+1}} y^{N_{2, n+1}}\right)=Z(x, y)\left\{P\left(N_{1, n}=N_{2, n}=0\right)\right. \\
& +E\left(x^{N_{1, n}} 1_{\left\{N_{1, n}>0, N_{2, n}=0\right\}}\right)\left[\alpha_{1}^{*} \tilde{P}_{0 /\{1\}}+\frac{\alpha_{1}^{*} \tilde{P}_{1 /\{1\}}}{x}+\bar{\alpha}_{1}^{*}\right] \\
& +E\left(y^{N_{2, n}} 1_{\left\{N_{1, n}=0, N_{2, n+1}>0\right\}}\right)\left[\alpha_{2}^{*} \tilde{P}_{0 /\{2\}}+\frac{\alpha_{2}^{*} \tilde{P}_{2 /\{2\}}}{y}+\bar{\alpha}_{2}^{*}\right] \\
& +E\left(x^{N_{1, n}} y^{N_{2, n}} 1_{\left\{N_{1, n}>0, N_{2, n}>0\right\}}\right)\left[\frac{\alpha_{1} \bar{\alpha}_{2} P_{1 /\{1\}}}{x}+\frac{\alpha_{2} \bar{\alpha}_{1} P_{2 /\{2\}}}{y}\right. \\
& +\alpha_{1} \bar{\alpha}_{2} P_{0 /\{1\}}+\alpha_{2} \bar{\alpha}_{1} P_{0 /\{2\}}+\bar{\alpha}_{1} \bar{\alpha}_{2} \\
& \left.\left.+\alpha_{1} \alpha_{2}\left(P_{0 /\{1,2\}}+\frac{P_{1 /\{1,2\}}}{x}+\frac{P_{2 /\{1,2\}}}{y}+\frac{P_{1,2 /\{1,2\}}}{x y}\right)\right]\right\},
\end{aligned}
$$

where $1_{\{F\}}$ denotes the indicator function of the event $F$. Using (5) we obtain after some algebra the following functional equation,

$$
\begin{aligned}
R(x, y) H(x, y)=A(x, y) H(x, 0) & +B(x, y) H(0, y) \\
& +C(x, y) H(0,0),
\end{aligned}
$$

where,

$$
\begin{aligned}
R(x, y)= & Z^{-1}(x, y)-1+\alpha_{1}\left(\bar{\alpha}_{2} P_{1 /\{1\}}+\alpha_{2} P_{1 /\{1,2\}}\right) \\
& \times\left(1-\frac{1}{x}\right)+\alpha_{2}\left(\bar{\alpha}_{1} P_{2 /\{2\}}+\alpha_{1} P_{2 /\{1,2\}}\right)\left(1-\frac{1}{y}\right) \\
& +\alpha_{1} \alpha_{2} P_{1,2 /\{1,2\}}\left(1-\frac{1}{x y}\right) \\
A(x, y)= & \alpha_{2}\left(\bar{\alpha}_{1} P_{2 /\{2\}}+\alpha_{1} P_{2 /\{1,2\}}\right)\left(1-\frac{1}{y}\right)+d_{1}\left(1-\frac{1}{x}\right) \\
& +\alpha_{1} \alpha_{2} P_{1,2 /\{1,2\}}\left(1-\frac{1}{x y}\right) \\
B(x, y)= & \alpha_{1}\left(\bar{\alpha}_{2} P_{1 /\{1\}}+\alpha_{2} P_{1 /\{1,2\}}\right)\left(1-\frac{1}{x}\right)+d_{2}\left(1-\frac{1}{y}\right) \\
& +\alpha_{1} \alpha_{2} P_{1,2 /\{1,2\}}\left(1-\frac{1}{x y}\right) \\
C(x, y)= & d_{2}\left(\frac{1}{y}-1\right)+d_{1}\left(\frac{1}{x}-1\right) \\
& +\alpha_{1} \alpha_{2} P_{1,2 /\{1,2\}}\left(\frac{1}{x y}-1\right)
\end{aligned}
$$

Our primary concern in order to obtain expressions for the average queueing delay is to solve the functional equation (6).
However, some interesting relations can be obtained directly. Indeed, setting $y=1$, dividing by $x-1$ and taking $x \rightarrow 1$ in (6), and vice versa, yield the following "conservation of flow" relations:

$$
\begin{aligned}
\lambda_{1}= & \alpha_{1} \widehat{\alpha}_{2}(1-H(0,1)-H(1,0)+H(0,0)) \\
& +\alpha_{1}^{*} \tilde{P}_{1 /\{1\}}(H(1,0)-H(0,0)), \\
\lambda_{2}= & \alpha_{2} \widehat{\alpha}_{1}(1-H(0,1)-H(1,0)+H(0,0)) \\
& +\alpha_{2}^{*} \tilde{P}_{2 /\{2\}}(H(0,1)-H(0,0)) .
\end{aligned}
$$

In the following, the analysis is distinguished in two cases:

1) For $\frac{\alpha_{1} \widehat{\alpha}_{2}}{\alpha_{1}^{*} \hat{P}_{1 /\{1\}}}+\frac{\alpha_{2} \widehat{\alpha}_{1}}{\alpha_{2}^{*} \tilde{P}_{2 /\{2\}}}=1$, (7) yields $H(0,0)=1-$ $\frac{\lambda_{1}}{\alpha_{1}^{*} \tilde{P}_{1 /\{1\}}}-\frac{\lambda_{2}}{\alpha_{2}^{*} \tilde{P}_{2 /\{2\}}}=1-\rho$.

2) In case $\frac{\alpha_{1} \widehat{\alpha}_{2}}{\alpha_{1}^{*} \tilde{P}_{1 /\{1\}}}+\frac{\alpha_{2} \widehat{\alpha}_{1}}{\alpha_{2}^{*} \hat{P}_{2 /\{2\}}} \neq 1$, (7) yields

$$
\begin{aligned}
& H(1,0)=\frac{\alpha_{1} \widehat{\alpha}_{2}\left(\lambda_{2}-\alpha_{2}^{*} \tilde{P}_{2 /\{2\}}\right)-\lambda_{1} \widehat{d}_{2}-\alpha_{1}^{*} \tilde{P}_{1 /\{1\}} \widehat{d}_{2} H(0,0)}{d_{1} d_{2}-\alpha_{1} \widehat{\alpha}_{2} \alpha_{2} \widehat{\alpha}_{1}}, \\
& H(0,1)=\frac{\alpha_{2} \widehat{\alpha}_{1}\left(\lambda_{1}-\alpha_{1}^{*} \tilde{P}_{1 /\{1\}}\right)-\lambda_{2} \widehat{d}_{1}-\alpha_{2}^{*} \tilde{P}_{2 /\{2\}} \widehat{d}_{1} H(0,0)}{d_{1} d_{2}-\alpha_{1} \widehat{\alpha}_{2} \alpha_{2} \widehat{\alpha}_{1}} .
\end{aligned}
$$

In the following we summarize the technical steps that we have to follow in order to obtain expressions for the queueing delay at each user node. The key element is the solution of the functional equation (6). The solution of (6) will provide the function $H(x, y)$. However, we firstly have to derive the boundary functions $H(x, 0), H(0, y)$, as well as $H(0,0)$. This is done using the theory of boundary value problems [36], [39].

Step 1.: From the functional equation (6), we prove that $H(x, 0)$ and $H(0, y)$ satisfy certain boundary value problems of Riemann-Carleman type [39], i.e., with boundary conditions on closed curves. These curves are studied in Lemma IV.3. The proof of this lemma (Appendix C) requires the investigation of the kernel $R(x, y)$ (see Section IV-A). All the required results are given in Lemmas IV.1, IV.2 (the proof of Lemma IV.1 is given in the Appendix B). The functions $H(x, 0), H(0, y)$ are analytic inside the unit disc, but they might have poles in the region bounded by the unit disc and these closed curves. The position of these poles are investigated in the Appendix D. Having in mind these poles, the boundary functions admit analytic continuations in the whole interiors of the curves above; see also Chapter 3 in [39]. Then, we have to obtain the precise boundary conditions on these curves. This is done in Subsections V-A, V-B; see (11), (16) respectively.

Step 2.: Next we conformally transform these problems into boundary value problems of Riemann-Hilbert type on the unit disc; see [36]. This conversion is motivated by the fact that the latter problems are more usual and by far more treated in the literature. It is done using conformal mappings in Subsections V-A, V-B; see (12).

Step 3.: Finally we solve these new problems and we deduce an explicit integral representation of the unknown boundary functions. This will conclude Subsections V-A, V-B; see (14), (18) respectively.

\section{A. Analysis of the kernel}

In the following we consider the kernel equation $R(x, y)=$ 0 and provide some important properties. We focus on a subclass of MPR channels, the so called "capture" channels, 
i.e., $P_{1,2 /\{1,2\}}=0$ (at most one user has a successful packet transmission even if many users transmit in that slot [45], [46]). Note that,

$$
R(x, y)=a(x) y^{2}+b(x) y+c(x)=\widehat{a}(y) x^{2}+\widehat{b}(y) x+\widehat{c}(y),
$$

where, $a(x)=\lambda_{2} x\left(\lambda_{1}(x-1)-1\right), b(x)=x\left(\lambda+\lambda_{1} \lambda_{2}+\right.$ $\left.\alpha_{1} \widehat{\alpha}_{2}+\alpha_{2} \widehat{\alpha}_{1}\right)-\alpha_{1} \widehat{\alpha}_{2}-\lambda_{1}\left(1+\lambda_{2}\right) x^{2}, c(x)=-\alpha_{2} \widehat{\alpha}_{1} x, \widehat{a}(y)=$ $\lambda_{1} y\left(\lambda_{2}(y-1)-1\right), \widehat{b}(y)=y\left(\lambda+\lambda_{1} \lambda_{2}+\alpha_{1} \widehat{\alpha}_{2}+\alpha_{2} \widehat{\alpha}_{1}\right)-\alpha_{2} \widehat{\alpha}_{1}-$ $\lambda_{2}\left(1+\lambda_{1}\right) y^{2}, \widehat{c}(y)=-\alpha_{1} \widehat{\alpha}_{2} y$. The roots of $R(x, y)=0$ are $X_{ \pm}(y)=\frac{-\widehat{b}(y) \pm \sqrt{D_{y}(y)}}{2 \widehat{a}(y)}, Y_{ \pm}(x)=\frac{-b(x) \pm \sqrt{D_{x}(x)}}{2 a(x)}$, where $D_{y}(y)=\widehat{b}(y)^{2}-4 \widehat{a}(y) \widehat{c}(y), D_{x}(x)=b(x)^{2}-4 a(x) c(x)$.

We proceed with Lemmas IV.1, IV.2, IV.3 that provide information about the kernel equation, and are important for the solution of (6). The proofs of Lemmas IV.1 and IV.3 are given in Appendixes $\mathrm{B}$ and $\mathrm{C}$ respectively.

Lemma IV.1. For $|y|=1, y \neq 1$, the kernel equation $R(x, y)=0$ has exactly one root $x=X_{0}(y)$ such that $\left|X_{0}(y)\right|<1$. For $\lambda_{1}<\alpha_{1} \widehat{\alpha}_{2}, X_{0}(1)=1$. Similarly, we can prove that $R(x, y)=0$ has exactly one root $y=Y_{0}(x)$, such that $\left|Y_{0}(x)\right| \leq 1$, for $|x|=1$.

Lemma IV.2. The algebraic function $Y(x)$, defined by $R(x, Y(x))=0$, has four real branch points $0<x_{1}<x_{2} \leq$ $1<x_{3}<x_{4}<\frac{1+\lambda_{1}}{\lambda_{1}}$. Moreover, $D_{x}(x)<0, x \in\left(x_{1}, x_{2}\right) \cup$ $\left(x_{3}, x_{4}\right)$ and $D_{x}(x)<0, x \in\left(-\infty, x_{1}\right) \cup\left(x_{2}, x_{3}\right) \cup\left(x_{4}, \infty\right)$. Similarly, $X(y)$, defined by $R(X(y), y)=0$, has four real branch points $0 \leq y_{1}<y_{2} \leq 1<y_{3}<y_{4}<\frac{1+\lambda_{2}}{\lambda_{2}}$. Moreover, $D_{x}(y)<0, y \in\left(y_{1}, y_{2}\right) \cup\left(y_{3}, y_{4}\right)$ and $D_{x}(y)>0$, $y \in\left(-\infty, y_{1}\right) \cup\left(y_{2}, y_{3}\right) \cup\left(y_{4}, \infty\right)$.

Proof. The proof is based on simple algebraic arguments and further details are omitted.

To ensure the continuity of the two valued function $Y(x)$ (resp. $X(y)$ ) we consider the cut planes: $\tilde{\tilde{C}}_{x}=\mathbb{C}_{x}-\left(\left[x_{1}, x_{2}\right] \cup\right.$ $\left.\left[x_{3}, x_{4}\right]\right), \tilde{\mathbf{C}}_{y}=\mathbb{C}_{y}-\left(\left[y_{1}, y_{2}\right] \cup\left[y_{3}, y_{4}\right]\right)$, where $\mathbb{C}_{x}, \mathbb{C}_{y}$ the complex planes of $x, y$, respectively. In $\tilde{\mathrm{C}}_{x}$ (resp. $\tilde{\mathrm{C}}_{y}$ ), denote by $Y_{0}(x)$ (resp. $X_{0}(y)$ ) the zero of $R(x, Y(x))=0$ (resp. $R(X(y), y)=0$ ) with the smallest modulus, and $Y_{1}(x)$ (resp. $\left.X_{1}(y)\right)$ the other one.

The following lemma shows that the mappings $Y(x), X(y)$, for $x \in\left[x_{1}, x_{2}\right], y \in\left[y_{1}, y_{2}\right]$ respectively, give rise to the smooth and closed contours $\mathcal{L}, \mathcal{M}$ respectively. Moreover, we also give exact representations of these contours (see Appendix C for the proof).

Lemma IV.3. 1) For $y \in\left[y_{1}, y_{2}\right]$, the algebraic function $X(y)$ lies on a closed contour $\mathcal{M}$, which is symmetric with respect to the real line and defined by $|x|^{2}=m(\operatorname{Re}(x))$, $m(\delta)=\frac{\alpha_{1} \widehat{\alpha}_{2}}{\lambda_{1}\left(1+\lambda_{2}-\lambda_{2} \zeta(\delta)\right)}$, and $|x|^{2} \leq \frac{\alpha_{1} \widehat{\alpha}_{2}}{\lambda_{1}\left(1+\lambda_{2}-\lambda_{2} y_{2}\right)}$, where, $\zeta(\delta)=\frac{k(\delta)-\sqrt{k^{2}(\delta)-4 \alpha_{2} \widehat{\alpha}_{1}\left(\lambda_{2}\left(1+\lambda_{1}(1-2 \delta)\right)\right)}}{2 \lambda_{2}\left(1+\lambda_{1}(1-2 \delta)\right)}$, $k(\delta):=\lambda+\lambda_{1} \lambda_{2}+\alpha_{1} \widehat{\alpha}_{2}+\alpha_{2} \widehat{\alpha}_{1}-2 \lambda_{1}\left(1+\lambda_{2}\right) \delta$. Set $\beta_{0}:=\sqrt{\frac{\alpha_{1} \widehat{\alpha}_{2}}{\lambda_{1}\left(1+\lambda_{2}-\lambda_{2} y_{2}\right)}}, \beta_{1}=-\sqrt{\frac{\alpha_{1} \widehat{\alpha}_{2}}{\lambda_{1}\left(1+\lambda_{2}-\lambda_{2} y_{1}\right)}}$ the extreme right and left point of $\mathcal{M}$, respectively.

2) For $x \in\left[x_{1}, x_{2}\right]$, the algebraic function $Y(x)$ lies on a closed contour $\mathcal{L}$, which is symmetric with respect to the real line and defined by $|y|^{2}=v(\operatorname{Re}(y)), v(\delta)=$ $\frac{\alpha_{2} \widehat{\alpha}_{1}}{\lambda_{2}\left(1+\lambda_{1}-\lambda_{1} \theta(\delta)\right)},|y|^{2} \leq \frac{\alpha_{2} \widehat{\alpha}_{1}}{\lambda_{2}\left(1+\lambda_{1}-\lambda_{1} x_{2}\right)}$. where $\theta(\delta)=$ $\frac{l(\delta)-\sqrt{l^{2}(\delta)-4 \alpha_{1} \widehat{\alpha}_{2}\left(\lambda_{1}\left(1+\lambda_{2}(1-2 \delta)\right)\right)}}{2 \lambda_{1}\left(1+\lambda_{2}(1-2 \delta)\right)}, l(\delta):=\lambda+\lambda_{1} \lambda_{2}+$ $\alpha_{1} \widehat{\alpha}_{2}+\alpha_{2} \widehat{\alpha}_{1}-2 \lambda_{2}\left(1+\lambda_{1}\right) \delta$. Set $\eta_{0}:=\sqrt{\frac{\alpha_{2} \widehat{\alpha}_{1}}{\lambda_{2}\left(1+\lambda_{1}-\lambda_{1} x_{2}\right)}}$, $\eta_{1}=-\sqrt{\frac{\alpha_{2} \widehat{\alpha}_{1}}{\lambda_{2}\left(1+\lambda_{1}-\lambda_{1} x_{1}\right)}}$ the extreme right and left point of $\mathcal{L}$, respectively.

\section{THE BOUNDARY VALUE PROBLEMS}

Based on a relation between the transmission probabilities of the users, we distinguish the analysis in two cases, which differ both from the modeling and the technical point of view (see Section IV). In this section we consider the case where $P_{1,2 /\{1,2\}}=0$ (i.e., the capture channel).

\section{A. A Dirichlet boundary value problem}

Assume now that $\frac{\alpha_{1} \widehat{\alpha}_{2}}{\alpha_{1}^{*} \tilde{P}_{1 /\{1\}}}+\frac{\alpha_{2} \widehat{\alpha}_{1}}{\alpha_{2}^{*} \tilde{P}_{2 /\{2\}}}=1$. Then, $A(x, y)=$ $\frac{d_{1}}{\alpha_{1} \widehat{\alpha}_{2}} B(x, y) \Leftrightarrow A(x, y)=\frac{\alpha_{2} \widehat{\alpha}_{1}}{d_{2}} B(x, y)$. Therefore, for $y \in$ $D_{y}=\left\{y \in C_{y}:|y| \leq 1,\left|X_{0}(y)\right| \leq 1\right\}$ we have

$$
\alpha_{2} \widehat{\alpha}_{1} H\left(X_{0}(y), 0\right)+d_{2} H(0, y)+\frac{\alpha_{2} \widehat{\alpha}_{1} C\left(X_{0}(y), y\right)}{A\left(X_{0}(y), y\right)}(1-\rho)=0 \text {. }
$$

For $y \in D_{y}-\left[y_{1}, y_{2}\right]$ both $H\left(X_{0}(y), 0\right), H(0, y)$ are analytic and the right-hand side can be analytically continued up to the slit $\left[y_{1}, y_{2}\right]$, or equivalently for $x \in \mathcal{M}$,

$$
\alpha_{2} \widehat{\alpha}_{1} H(x, 0)+d_{2} H\left(0, Y_{0}(x)\right)+\frac{\alpha_{2} \widehat{\alpha}_{1} C\left(x, Y_{0}(x)\right)}{A\left(x, Y_{0}(x)\right)}(1-\rho)=0 .
$$

Then, multiplying both sides of (10) by the imaginary complex number $i$, and noticing that $H\left(0, Y_{0}(x)\right)$ is real for $x \in \mathcal{M}$, since $Y_{0}(x) \in\left[y_{1}, y_{2}\right]$, we have for $x \in \mathcal{M}$,

$$
R e(i H(x, 0))=\operatorname{Re}\left(-i \frac{C\left(x, Y_{0}(x)\right)}{A\left(x, Y_{0}(x)\right)}\right)(1-\rho) .
$$

In the following we have to check for poles of $H(x, 0)$ in $S_{x}:=G_{\mathcal{M}} \cap \bar{D}_{x}^{c}$, where $G_{\mathcal{U}}$ be the interior domain bounded by $\mathcal{U}$, and $D_{x}=\{x:|x|<1\}, \bar{D}_{x}=\{x:|x| \leq 1\}$, $\bar{D}_{x}^{c}=\{x:|x|>1\}$. These poles, if exist, they coincide with the zeros of $A\left(x, Y_{0}(x)\right)$ in $S_{x}$ (see Appendix D). In order to solve (11), we must first conformally transform the problem from $\mathcal{M}$ to the unit circle $\mathcal{C}$. Let the conformal mapping, $z=$ $\gamma(x): G_{\mathcal{M}} \rightarrow G_{\mathcal{C}}$, and its inverse $x=\gamma_{0}(z): G_{\mathcal{C}} \rightarrow G_{\mathcal{M}}$.

Then, we have the following problem: Find a function $\tilde{T}(z)=H\left(\gamma_{0}(z), 0\right)$ regular for $z \in G_{\mathcal{C}}$, and continuous for $z \in \mathcal{C} \cup G_{\mathcal{C}}$ such that, $\operatorname{Re}(i \tilde{T}(z))=w\left(\gamma_{0}(z)\right), z \in \mathcal{C}$. To proceed, we need a representation of $\mathcal{M}$ in polar coordinates, i.e., $\mathcal{M}=\{x: x=\rho(\phi) \exp (i \phi), \phi \in[0,2 \pi]\}$. This procedure is described in detail in [36]. In the following we summarize the basic steps: Since $0 \in G_{\mathcal{M}}$, for each $x \in \mathcal{M}$, a relation between its absolute value and its real part is given by $|x|^{2}=m(R e(x)$ ) (see Lemma IV.3). Given the angle $\phi$ of some point on $\mathcal{M}$, the real part of this point, say $\delta(\phi)$, is the solution of $\delta-\cos (\phi) \sqrt{m(\delta)}, \phi \in[0,2 \pi]$. Since $\mathcal{M}$ is a smooth, egg-shaped contour, the solution is unique. Clearly, $\rho(\phi)=\frac{\delta(\phi)}{\cos (\phi)}$, and the parametrization of $\mathcal{M}$ in polar coordinates is fully specified; [36]. Then, the mapping from $z \in G_{\mathcal{C}}$ to $x \in G_{\mathcal{M}}$, where $z=e^{i \phi}$ and $x=\rho(\psi(\phi)) e^{i \psi(\phi)}$, 
satisfying $\gamma_{0}(0)=0, \gamma_{0}(z)=\overline{\gamma_{0}(z)}$ is uniquely determined by,

$$
\begin{aligned}
& \gamma_{0}(z)=\quad z \exp \left[\frac{1}{2 \pi} \int_{0}^{2 \pi} \log \{\rho(\psi(\omega))\} \frac{e^{i \omega}+z}{e^{i \omega}-z} d \omega\right],|z|<1, \\
& \psi(\phi)=\phi-\int_{0}^{2 \pi} \log \{\rho(\psi(\omega))\} \cot \left(\frac{\omega-\phi}{2}\right) d \omega, 0 \leq \phi \leq 2 \pi,
\end{aligned}
$$

i.e., $\psi($.$) is uniquely determined as the solution of a$ Theodorsen integral equation with $\psi(\phi)=2 \pi-\psi(2 \pi-\phi)$. Due to the correspondence-boundaries theorem, $\gamma_{0}(z)$ is continuous in $\mathcal{C} \cup G_{\mathcal{C}}$.

If $H(x, 0)$ has no poles in $S_{x}$, the solution of the problem defined in (11) is:

$$
H(x, 0)=-\frac{1-\rho}{2 \pi} \int_{|t|=1} f(t) \frac{t+\gamma(x)}{t-\gamma(x)} \frac{d t}{t}+K, x \in \mathcal{M},
$$

where $f(t)=\operatorname{Re}\left(-i \frac{C\left(\gamma_{0}(t), Y_{0}\left(\gamma_{0}(t)\right)\right)}{A\left(\gamma_{0}(t), Y_{0}\left(\gamma_{0}(t)\right)\right)}\right) . K$ is a constant that can be obtained by setting $x=0 \in G_{\mathcal{M}}$ in (13), and using the fact that $H(0,0)=1-\rho, \gamma(0)=0$. If $H(x, 0)$ has a pole, it will be $x=\bar{x}$ (see Appendix D), and we still have a Dirichlet problem for the function $(x-\bar{x}) H(x, 0)$.

Following the discussion above, $K=(1-$ $\rho)\left(1+\frac{1}{2 \pi} \int_{|t|=1} f(t) \frac{d t}{t}\right)$. Setting $t=e^{i \phi}, \gamma_{0}\left(e^{i \phi}\right)=$ $\rho(\psi(\phi)) e^{i \psi(\phi)}$, we obtain after some algebra,

$$
\frac{f\left(e^{i \phi}\right)=}{\rho(\psi(\phi))\left\{\left[\alpha_{2} \widehat{\alpha}_{1}\left(1-Y_{0}^{-1}\left(\gamma_{0}\left(e^{i \phi}\right)\right)\right)+d_{1}\left(1-\frac{\cos (\psi(\phi))}{\rho(\psi(\phi))}\right)\right]^{2}+\left(d_{1} \frac{\sin (\psi(\phi))}{\rho(\psi(\phi))}\right)^{2}\right\}},
$$

which is an odd function of $\phi$. Thus, $K=1-\rho$. Substituting back in (13) we obtain for $x \in G_{\mathcal{M}}$,

$$
H(x, 0)=(1-\rho)\left\{1+\frac{2 \gamma(x) i}{\pi} \int_{0}^{\pi} \frac{f\left(e^{i \phi}\right) \sin (\phi)}{1-2 \gamma(x) \cos (\phi)-\gamma(x)^{2}} d \phi\right\} .
$$

A detailed numerical approach in order to obtain the inverse mapping $\gamma(x)$ is presented in the seminal book [36]. Similarly, we can determine $H(0, y)$ by solving another Dirichlet boundary value problem on the closed contour $\mathcal{L}$. Then, using equation (6) we uniquely obtain $H(x, y)$.

\section{B. A homogeneous Riemann-Hilbert boundary value problem}

We now assume that $\frac{\alpha_{1} \widehat{\alpha}_{2}}{\alpha_{1}^{*} \tilde{P}_{1 /\{1\}}}+\frac{\alpha_{2} \widehat{\alpha}_{1}}{\alpha_{2}^{*} \tilde{P}_{2 /\{2\}}} \neq 1$, and consider the following transformation:

$$
\begin{aligned}
G(x) & :=H(x, 0)+\frac{\alpha_{1}^{*} \tilde{P}_{1 /\{1\}} d_{2} H(0,0)}{d_{1} d_{2}-\alpha_{1} \widehat{\alpha}_{2} \alpha_{2} \widehat{\alpha}_{1}}, \\
L(y) & :=H(0, y)+\frac{\alpha_{2}^{*} \tilde{P}_{2 /\{2\}} d_{1} H(0,0)}{d_{1} d_{2}-\alpha_{1} \widehat{\alpha}_{2} \alpha_{2} \widehat{\alpha}_{1}} .
\end{aligned}
$$

Then, for $y \in D_{y}$, equation (6) is rewritten as $A\left(X_{0}(y), y\right) G\left(X_{0}(y)\right)=-B\left(X_{0}(y), y\right) L(y)$. For $y \in D_{y}-$ $\left[y_{1}, y_{2}\right]$ both $G\left(X_{0}(y)\right), L(y)$ are analytic and the right-hand side can be analytically continued up to the slit $\left[y_{1}, y_{2}\right]$, or equivalently, we have for $x \in \mathcal{M}$,

$$
A\left(x, Y_{0}(x)\right) G(x)=-B\left(x, Y_{0}(x)\right) L\left(Y_{0}(x)\right) .
$$

Clearly, $G(x)$ is holomorphic in $D_{x}$, continuous in $\bar{D}_{x}$. However, $G(x)$ might has poles, based on the values of the system parameters in $S_{x}=G_{\mathcal{M}} \cap \bar{D}_{x}^{c}$. These poles (if exist) coincide with the zeros of $A\left(x, Y_{0}(x)\right)$ in $S_{x}$; see Appendix D. For $y \in\left[y_{1}, y_{2}\right]$, let $X_{0}(y)=x \in \mathcal{M}$ and notice that $Y_{0}\left(X_{0}(y)\right)=y$ so that $y=Y_{0}(x)$ (note that $\left.B\left(x, Y_{0}(x)\right) \neq 0, x \in \mathcal{M}\right)$. Taking into account the possible poles of $G(x)$, and noticing that $L\left(Y_{0}(x)\right)$ is real for $x \in \mathcal{M}$, since $Y_{0}(x) \in\left[y_{1}, y_{2}\right]$,

$$
\begin{gathered}
\operatorname{Re}[i U(x) \tilde{G}(x)]=0, x \in \mathcal{M}, \\
U(x)=\frac{A\left(x, Y_{0}(x)\right)}{(x-\bar{x})^{r} B\left(x, Y_{0}(x)\right)}, \tilde{G}(x)=(x-\bar{x})^{r} G(x),
\end{gathered}
$$

where $r=0,1$, whether $\bar{x}$ is zero or not of $A\left(x, Y_{0}(x)\right)$ in $S_{x}$. Thus, $\tilde{G}(x)$ is regular for $x \in G_{\mathcal{M}}$, continuous for $x \in$ $\mathcal{M} \cup G_{\mathcal{M}}$, and $U(x)$ is a non-vanishing function on $\mathcal{M}$. We must first conformally transform the problem (16) from $\mathcal{M}$ to the unit circle $\mathcal{C}$. Let the conformal mapping $z=\gamma(x)$ : $G_{\mathcal{M}} \rightarrow G_{\mathcal{C}}$, and its inverse given by $x=\gamma_{0}(z): G_{\mathcal{C}} \rightarrow G_{\mathcal{M}}$.

Then, the Riemann-Hilbert problem formulated in (16) is reduced to the following: Find a function $F(z):=$ $\tilde{G}\left(\gamma_{0}(z)\right)$, regular in $G_{\mathcal{C}}$, continuous in $G_{\mathcal{C}} \cup \mathcal{C}$ such that, $\operatorname{Re}\left[i U\left(\gamma_{0}(z)\right) F(z)\right]=0, z \in \mathcal{C}$.

A crucial step in the solution of the problem defined by (16) is the determination of the index $\chi=\frac{-1}{\pi}[\arg \{U(x)\}]_{x \in \mathcal{M}}$, where $[\arg \{U(x)\}]_{x \in \mathcal{M}}$, denotes the variation of the argument of the function $U(x)$ as $x$ moves along the closed contour $\mathcal{M}$ in the positive direction, provided that $U(x) \neq 0, x \in \mathcal{M}$. Following the lines in [39] we have the next Lemma.

Lemma V.1. 1) If $\lambda_{2}<\alpha_{2} \widehat{\alpha}_{1}$, then $\chi=0$ is equivalent to

$$
\begin{aligned}
& \left.\frac{d A\left(x, Y_{0}(x)\right)}{d x}\right|_{x=1}<0 \Leftrightarrow \lambda_{1}<\alpha_{1}^{*} \tilde{P}_{1 /\{1\}}+\widehat{d}_{1} \frac{\lambda_{2}}{\alpha_{2} \widehat{\alpha}_{1}}, \\
& \left.\frac{d B\left(X_{0}(y), y\right)}{d y}\right|_{y=1}<0 \Leftrightarrow \lambda_{2}<\alpha_{2}^{*} \tilde{P}_{2 /\{2\}}+\widehat{d}_{2} \frac{\lambda_{1}}{\alpha_{1} \widehat{\alpha}_{2}} .
\end{aligned}
$$

2) If $\lambda_{2} \geq \alpha_{2} \widehat{\alpha}_{1}, \chi=0$ is equivalent to $\left.\frac{d B\left(X_{0}(y), y\right)}{d y}\right|_{y=1}<$ $0 \Leftrightarrow \lambda_{2}<\alpha_{2}^{*} \tilde{P}_{2 /\{2\}}+\widehat{d}_{2} \frac{\lambda_{1}}{\alpha_{1} \widehat{\alpha}_{2}}$.

Thus, under stability conditions (see Lemma III.1) the problem in (16) has a unique solution,

$$
\begin{aligned}
H(x, 0)= & W(x-\bar{x})^{r} \exp \left[\frac{1}{2 i \pi} \int_{|t|=1} \frac{\log \{J(t)\}}{t-\gamma(x)} d t\right] \\
& -\frac{\alpha_{1}^{*} \tilde{P}_{1 /\{1\}} d_{2} H(0,0)}{d_{1} d_{2}-\alpha_{1} \widehat{\alpha}_{2} \alpha_{2} \widehat{\alpha}_{1}}, x \in G_{\mathcal{M}},
\end{aligned}
$$

where $W$ is a constant and $J(t)=\frac{\overline{U_{1}(t)}}{U_{1}(t)}, U_{1}(t)=U\left(\gamma_{0}(t)\right)$, $|t|=1$. Setting $x=0$ in (17) we derive a relation between $W$ and $H(0,0)$. Then, for $x=1 \in G_{\mathcal{M}}$, and using the first in (8) we can obtain $W$ and $H(0,0)$. Substituting back in (17) we obtain for $x \in G_{\mathcal{M}}$,

$$
\begin{aligned}
& H(x, 0)=\frac{\lambda_{1} d_{2}-\alpha_{1} \widehat{\alpha}_{2}\left(\lambda_{2}-\alpha_{2}^{*} \tilde{P}_{2 /\{2\}}\right)}{\left(\alpha_{1} \widehat{\alpha}_{2} \alpha_{2} \widehat{\alpha}_{1}-d_{1} d_{2}\right)(\bar{x}-1)^{r}}\left\{(\bar{x}-x)^{r}\right. \\
& \times \exp \left[\frac{\gamma(x)-\gamma(1)}{2 i \pi} \int_{|t|=1} \frac{\log \{J(t)\}}{(t-\gamma(x))(t-\gamma(1))} d t\right] \\
& \left.+\frac{\alpha_{1}^{*} \tilde{P}_{1 /\{1\}} d_{2}(\bar{x})^{r}}{\alpha_{1} \widehat{\alpha}_{2} \alpha_{2}^{*} \tilde{P}_{2 /\{2\}}} \exp \left[\frac{-\gamma(1)}{2 i \pi} \int_{|t|=1} \frac{\log \{J(t)\}}{t(t-\gamma(1))} d t\right]\right\} .
\end{aligned}
$$

Similarly, we can determine $H(0, y)$ by solving another Dirichlet boundary value problem on the closed contour $\mathcal{L}$. Then, using the functional equation (6) we uniquely obtain $H(x, y)$.

\section{Expected Number of Packets and Average Delay}

In the following we derive formulas for the expected number of packets and the average delay at each user node in steady 
state, say $L_{i}$ and $D_{i}, i=1,2$, respectively. Denote by $H_{1}(x, y), H_{2}(x, y)$ the derivatives of $H(x, y)$ with respect to $x$ and $y$ respectively. Then, $L_{i}=H_{i}(1,1)$, and using Little's law $D_{i}=H_{i}(1,1) / \lambda_{i}, i=1,2$. Using equations (6) and (7) we derive

$$
L_{1}=\frac{\lambda_{1}+d_{1} H_{1}(1,0)}{\alpha_{1} \widehat{\alpha}_{2}}, \quad L_{2}=\frac{\lambda_{2}+d_{2} H_{2}(0,1)}{\alpha_{2} \widehat{\alpha}_{1}} .
$$

We only focus on $L_{1}, D_{1}$ (similarly we obtain $L_{2}, D_{2}$ ). When $\frac{\alpha_{1} \widehat{\alpha}_{2}}{\alpha_{1}^{*} \tilde{P}_{1 /\{1\}}}+\frac{\alpha_{2} \widehat{\alpha}_{1}}{\alpha_{2}^{*} \tilde{P}_{2 /\{2\}}} \neq 1$, using (18),

$$
\begin{aligned}
& H_{1}(1,0)=\frac{\lambda_{1} d_{2}+\alpha_{1} \widehat{\alpha}_{2}\left(\alpha_{2}^{*} \tilde{P}_{2 /\{2\}}-\lambda_{2}\right)}{\alpha_{1} \widehat{\alpha}_{2} \alpha_{2} \widehat{\alpha}_{1}-d_{1} d_{2}} \\
& \times\left\{\frac{\gamma^{\prime}(1)}{2 \pi i} \int_{|t|=1} \frac{\log \{J(t)\}}{(t-\gamma(1))^{2}} d t+\frac{r}{1-\bar{x}} 1_{\{r=1\}}\right\} .
\end{aligned}
$$

Substituting (20) in (19) we obtain $L_{1}$, and dividing with $\lambda_{1}$, the average delay $D_{1}$. Note that the calculation of (12) requires the evaluation of integrals (12), and $\gamma(1), \gamma^{\prime}(1)$. For an efficient numerical procedure see [36], Chapter IV.1.

\section{Stability CONDitions: Extension to THE CASE OF $N=3$ USERS}

In the following, we provide sufficient and necessary conditions for the the case of $N=3$ users based on [15]. In particular we generalize the results in [15], by including the effect of capture channel as well as the queue-aware transmission policy. We accomplish this by means of a technique based on three simple observations: isolating a single queue from the system, applying Loynes' stability criteria for such an isolated queue, and using stochastic dominance and mathematical induction to verify the required stationarity assumptions in the Loynes' criterion. Below, we present an informal overview of the approach. First of all, we construct a modified system as follows. Let $P=(S, U)$ be a partition of $M=\{1,2,3\}$ such that users in $S \neq M$ operate exactly as in the original model, while users in $U$ are able to send packets even if their buffers are empty (i.e., dummy packets). Note that a system consisting of users in $S$ forms a smaller copy of the original system with slightly new transmission probabilities. Furthermore, it is easy to see that the modified system, stochastically dominates the queue lengths process in the original system; see [35], [15]. Thus, proving stability of such a dominant system - that is, the one under the partition $(S, U)$ - suffices for stability of the original system. To accomplish this, we prove stability conditions for users in $S$ by mathematical induction. Finally, the stability region for the original system is a union of stability regions obtained for every partition $P$; see Theorem 1 in [15]. As proved in [15], only partitions $P_{k}=\left(M_{k},\{k\}\right)$, where $M_{k}=M-\{k\}$ contribute to the final stability region.

\section{A. An alternative approach for the case of $N=2$ users}

We will first derive the stability region for the case of $N=2$ users in order to assist the analysis for the case of $N=3$ users. For such a case we consider the partitions $P_{1}=\left(M_{1},\{1\}\right)$, $P_{2}=\left(M_{2},\{2\}\right)$, where $M_{1}=\{2\}$ and $M_{2}=\{1\}$, and let $\mathcal{R}_{i}$ be stability region for the partition $P_{i}, i=1,2$. We will discuss in detail the construction of $\mathcal{R}_{1}$; similarly we can construct $\mathcal{R}_{2}$. Denote by $P_{\text {suc }}^{(i)}\left(M_{j}\right)$ the probability of a successful transmission from user $i$ in the dominant system $M_{j}$. Clearly,

$$
\begin{aligned}
P_{\text {suc }}^{(1)}\left(M_{1}\right)= & \alpha_{1}^{*} \tilde{P}_{1 /\{1\}} \operatorname{Pr}\left(N_{2}=0\right)+\alpha_{1}\left(\bar{\alpha}_{2} P_{1 /\{1\}}\right. \\
& \left.+\alpha_{2} P_{1 /\{1,2\}}\right) \operatorname{Pr}\left(N_{2}>0\right) \\
= & \alpha_{1}^{*} \tilde{P}_{1 /\{1\}}+d_{1} P\left(N_{2}>0\right), \\
P_{\text {suc }}^{(2)}\left(M_{1}\right)= & \alpha_{2}\left(\bar{\alpha}_{1} P_{2 / 2}+\alpha_{1} P_{2 /\{1,2\}}\right)=\alpha_{2} \widehat{\alpha}_{1} .
\end{aligned}
$$

For $\lambda_{2}<P_{\text {suc }}^{(2)}\left(M_{1}\right), \operatorname{Pr}\left(N_{2}>0\right)=\lambda_{2} /\left(\alpha_{2} \widehat{\alpha}_{1}\right)$, and hence $\mathcal{R}_{1}$ is obtained. Similarly, by considering $M_{2}$ we obtain $\mathcal{R}_{2}$. Note that the stability conditions are the same with those obtained in Theorem III.1.

\section{B. The case of $N=3$ users}

Here we consider the case of $N=3$ users, which is more intricate. We now have to investigate only three partitions $P_{i}=\left(M_{i},\{i\}\right)$, where $M_{1}=\{2,3\}, M_{2}=\{1,3\}$ and $M_{3}=\{1,2\}$, and only the first partition will be discussed in detail. As stated previously, the stability region $\mathcal{R}$ is the union of three regions $\mathcal{R}_{1}, \mathcal{R}_{2}$ and $\mathcal{R}_{3}$ each corresponding to $M_{1}, M_{2}$, and $M_{3}$, respectively.

To proceed, we have to make clear how the system operates: for convenience we assume that node $i, i=1,2,3$, transmits a packet to the common destination with probability $\alpha_{i}$ when the node $(i \bmod 3+1)$ is non empty, and with probability $\alpha_{i}^{*} \geq \alpha_{i}$ when the node $(i \bmod 3+1)$ is empty. We will present the derivation of $\mathcal{R}_{1}$. In the corresponding dominant system, the first user never empties. Note that such a system can be viewed as a two-user system with an additional user who creates interference (i.e., it transmits dummy packets when it is empty) to the other users. In order to proceed we will perform a similar analysis as in Section $\mathrm{V}$.

Let $F_{1}(y, z)$ be the generating function of $\left(N_{2, n}, N_{3, n}\right)$ with the first user being an interfering one (i.e., it never empties). Then, with a minor modification,

$$
\begin{aligned}
& \lambda_{2}=\alpha_{2} \widehat{\alpha}_{13}^{(2)}\left(1-F_{1}(0,1)\right)-d_{2}^{*}\left(F_{1}(1,0)-F_{1}(0,0)\right), \\
& \lambda_{3}=\alpha_{3} \widehat{\alpha}_{12}^{(3)}\left(1-F_{1}(1,0)\right)-d_{3}^{*}\left(F_{1}(0,1)-F_{1}(0,0)\right),
\end{aligned}
$$

where, for $k \neq i \neq j, k, i, j \in M=\{1,2,3\}$,

$$
\begin{aligned}
\widehat{\alpha}_{i j}^{(k)}= & \bar{\alpha}_{i} \bar{\alpha}_{j} P_{k /\{k\}}+\alpha_{i} \bar{\alpha}_{j} P_{k /\{k, i\}}+\alpha_{j} \bar{\alpha}_{i} P_{k /\{k, j\}} \\
& +\alpha_{i} \alpha_{j} P_{k /\{k, i, j\}}, \\
d_{2}^{*}= & \alpha_{2} \widehat{\alpha}_{13}^{(2)}-\alpha_{2}^{*} \bar{\alpha}_{12}, \\
d_{3}^{*}= & \alpha_{3}\left(\widehat{\alpha}_{12}^{(3)}-\bar{\alpha}_{13}^{*}\right), \\
\bar{\alpha}_{i j}= & \bar{\alpha}_{i} \tilde{P}_{j /\{j\}}+\alpha_{i} \tilde{P}_{j /\{i, j\}}, \\
\bar{\alpha}_{i j}^{*}= & \bar{\alpha}_{i}^{*} \tilde{P}_{j /\{j\}}+\alpha_{i}^{*} \tilde{P}_{j /\{i, j\}}, i, j \in M, i \neq j,
\end{aligned}
$$

and $P_{i / T}$ is the success probability of user $i$ when the transmitting users are in $T$ and all users have packets to send, $\tilde{P}_{i /\{T\}}$ is the success probability of user $i$ when the transmitting users are in $T$ and there is only one user $(\neq$ $i, 1)$ that is empty. Following [15], for $z_{2}, z_{3} \in\{0,1\}$, let $P_{1}\left(z_{2}, z_{3}\right)=P\left(\chi\left(\bar{N}_{2}\right)=z_{2}, \chi\left(\bar{N}_{3}\right)=z_{3}\right)$, with the first user be the interfering one, $\left(\bar{N}_{2}, \bar{N}_{3}\right)$ the queue lengths in the 
modified system and $\chi(k)=0$ for $k=0$ and $\chi(k)=1$ for $k \geq 1$. Then, from the analysis in section $\mathrm{V}$, we have

$$
\begin{aligned}
P_{1}(0,0)= & \frac{\alpha_{2} \widehat{\alpha}_{13}^{(2)}\left(\alpha_{3} \widehat{\alpha}_{12}^{(3)}-\lambda_{3}\right)+d_{3}^{*}\left(\lambda_{2}-\alpha_{2} \widehat{\alpha}_{13}^{(2)}\right)}{\alpha_{2} \widehat{\alpha}_{13}^{(2)} \alpha_{3} \bar{\alpha}_{13}^{*}} \\
& \times \exp \left[\frac{-\gamma(1)}{2 \pi i} \int_{|t|=1} \frac{\log \{J(t)\}}{t(t-\gamma(1))} d t\right],
\end{aligned}
$$

where $\gamma(x)$ is the inverse of a conformal mapping of the unit circle onto a curve $\mathcal{M}^{*}$ (see subsection IV-A). Note also that $P_{1}(1,0)=F_{1}(1,0)-F_{1}(0,0), P_{1}(0,1)=F_{1}(0,1)-F_{1}(0,0)$, $P_{1}(1,1)=1-F_{1}(1,0)-F_{1}(0,1)+F_{1}(0,0), P_{1}(0,0)=$ $F_{1}(0,0)$. Now, for $P_{1}=\left(M_{1},\{1\}\right)$, and after some algebra,

$$
\begin{array}{ll}
P_{\text {suc }}^{(1)}\left(M_{1}\right)= & \alpha_{1}^{*} \tilde{P}_{1 /\{1\}}^{\prime} P_{1}(0,0)+\alpha_{1} \bar{\alpha}_{21}^{*} P_{1}(1,0) \\
& +\alpha_{1}^{*} \bar{\alpha}_{31} P_{1}(0,1)+\alpha_{1} \widehat{\alpha}_{23}^{(1)} P_{1}(1,1), \\
P_{\text {suc }}^{(2)}\left(M_{1}\right)= & \alpha_{2} \widehat{\alpha}_{13}^{(2)}+F_{1}(1,0)\left(\alpha_{2}^{*} \bar{\alpha}_{12}-\alpha_{2} \widehat{\alpha}_{13}^{(2)}\right), \\
P_{\text {suc }}^{(3)}\left(M_{1}\right)= & \alpha_{3} \widehat{\alpha}_{12}^{(3)}+F_{1}(0,1) \alpha_{3}\left(\bar{\alpha}_{13}^{*}-\widehat{\alpha}_{12}^{(3)}\right),
\end{array}
$$

where $\tilde{P}_{i /\{i\}}^{\prime}$ is the success probability for a user $i$, when it is the only non-empty node.

Note that from (21), provided that $d_{2}^{*} d_{3}^{*}-\alpha_{2} \widehat{\alpha}_{13}^{(2)} \alpha_{3} \widehat{\alpha}_{12}^{(3)} \neq 0$,

$$
\begin{aligned}
& F_{1}(1,0)=\frac{\alpha_{2} \widehat{\alpha}_{13}^{(2)}\left(\alpha_{3} \widehat{\alpha}_{12}^{(3)}-\lambda_{3}\right)+d_{3}^{*}\left(\lambda_{2}-\alpha_{2} \widehat{\alpha}_{13}^{(2)}\right)+d_{3}^{*} \alpha_{2}^{*} \bar{\alpha}_{12} F_{1}(0,0)}{\alpha_{2} \widehat{\alpha}_{13}^{(2)} \alpha_{3} \widehat{\alpha}_{12}^{(3)}-d_{2}^{*} d_{3}^{*}}, \\
& F_{1}(0,1)=\frac{\alpha_{3} \widehat{\alpha}_{12}^{(3)}\left(\alpha_{2} \widehat{\alpha}_{13}^{(2)}-\lambda_{2}\right)+d_{2}^{*}\left(\lambda_{3}-\alpha_{3} \widehat{\alpha}_{12}^{(3)}\right)+d_{2}^{*} \alpha_{3} \bar{\alpha}_{13}^{*} F_{1}(0,0)}{\alpha_{2} \widehat{\alpha}_{13}^{(2)} \alpha_{3} \widehat{\alpha}_{12}^{(3)}-d_{2}^{*} d_{3}^{*}} .
\end{aligned}
$$

Therefore, after some simple but tedious calculations we have

$$
\begin{array}{r}
P_{s u c}^{(1)}\left(M_{1}\right)=P_{1}(0,0)\left\{\alpha_{1}^{*} \tilde{P}_{1 /\{1\}}^{\prime}-\alpha_{1} \bar{\alpha}_{21}^{*}-\alpha_{1}^{*} \bar{\alpha}_{31}-\alpha_{1} \widehat{\alpha}_{23}^{(1)}\right. \\
\left.+\frac{\left.\left(\alpha_{1} \bar{\alpha}_{21}^{*}-\alpha_{1} \widehat{\alpha}_{23}^{(1)}\right) d_{3}^{*} \alpha_{2}^{*} \bar{\alpha}_{12}+\left(\alpha_{1}^{*} \bar{\alpha}_{31}-\alpha_{1} \widehat{\alpha}_{23}^{(1)}\right) d_{2}^{*} \alpha_{3} \bar{\alpha}_{13}^{*}\right\}}{\alpha_{2} \widehat{\alpha}_{13}^{(2)} \alpha_{3} \widehat{\alpha}_{12}^{(3)}-d_{2}^{*} d_{3}^{*}}\right\} \\
+\frac{\left(\alpha_{3} \widehat{\alpha}_{12}^{(3)}-\lambda_{3}\right)\left[\alpha_{2} \widehat{\alpha}_{13}^{(2)}\left(\alpha_{1} \bar{\alpha}_{21}^{*}-\alpha_{1} \widehat{\alpha}_{23}^{(1)}\right)-d_{2}^{*}\left(\alpha_{1}^{*} \bar{\alpha}_{31}-\alpha_{1} \widehat{\alpha}_{23}^{(1)}\right)\right]}{\alpha_{2} \widehat{\alpha}_{13}^{(2)} \alpha_{3} \widehat{\alpha}_{12}^{(3)}-d_{2}^{*} d_{3}^{*}} \\
+\frac{\left(\alpha_{2} \widehat{\alpha}_{13}^{(2)}-\lambda_{2}\right)\left[\alpha_{3} \widehat{\alpha}_{12}^{(3)}\left(\alpha_{1}^{*} \bar{\alpha}_{31}-\alpha_{1} \widehat{\alpha}_{23}^{(1)}\right)-d_{3}^{*}\left(\alpha_{1} \bar{\alpha}_{21}^{*}-\alpha_{1} \widehat{\alpha}_{23}^{(1)}\right)\right]}{\alpha_{2} \widehat{\alpha}_{13}^{(2)} \alpha_{3} \widehat{\alpha}_{12}^{(3)}-d_{2}^{*} d_{3}^{*}} .
\end{array}
$$

Similarly, using (23) we can express $P_{\text {suc }}^{(2)}\left(M_{1}\right), P_{\text {suc }}^{(3)}\left(M_{1}\right)$ in terms of $F_{1}(0,0)$.

In summary, following [15] we have the following corollary.

Corollary VI.1. The system with three users is stable if and only if $\left(\lambda_{1}, \lambda_{2}, \lambda_{3}\right) \in \mathcal{R}=\mathcal{R}_{1} \cup \mathcal{R}_{2} \cup \mathcal{R}_{3}$, where

$$
\begin{aligned}
\mathcal{R}_{1}= & \left\{\left(\lambda_{1}, \lambda_{2}\right): \lambda_{1}<P_{s u c}^{(1)}\left(M_{1}\right), \lambda_{2}<\alpha_{2} \widehat{\alpha}_{13}^{(2)}+F_{1}(1,0)\right. \\
& \left.\times\left(\alpha_{2}^{*} \bar{\alpha}_{12}-\alpha_{2} \widehat{\alpha}_{13}^{(2)}\right), \lambda_{3}<\alpha_{3} \widehat{\alpha}_{12}^{(3)}+F_{1}(0,1) \alpha_{3}\left(\bar{\alpha}_{13}^{*}-\widehat{\alpha}_{12}^{(3)}\right)\right\}, \\
\mathcal{R}_{2}= & \left\{\left(\lambda_{1}, \lambda_{2}\right): \lambda_{1}<\alpha_{1} \widehat{\alpha}_{23}^{(1)}+F_{2}(1,0) \alpha_{1}\left(\bar{\alpha}_{21}^{*}-\widehat{\alpha}_{23}^{(1)}\right),\right. \\
& \left.\lambda_{2}<P_{\text {suc }}^{(2)}\left(M_{2}\right), \lambda_{3}<\alpha_{3} \widehat{\alpha}_{12}^{(3)}+F_{2}(0,1)\left(\alpha_{3}^{*} \bar{\alpha}_{23}-\alpha_{3} \widehat{\alpha}_{12}^{(3)}\right)\right\}, \\
\mathcal{R}_{3}= & \left\{\left(\lambda_{1}, \lambda_{2}\right): \lambda_{1}<\alpha_{1} \widehat{\alpha}_{23}^{(1)}+F_{3}(1,0)\left(\alpha_{1}^{*} \bar{\alpha}_{31}-\alpha_{1} \widehat{\alpha}_{23}^{(1)}\right),\right. \\
& \left.\lambda_{2}<\alpha_{2} \widehat{\alpha}_{13}^{(2)}+F_{3}(0,1) \alpha_{2}\left(\bar{\alpha}_{32}^{*}-\widehat{\alpha}_{13}^{(2)}\right), \lambda_{3}<P_{\text {suc }}^{(3)}\left(M_{3}\right)\right\},
\end{aligned}
$$

where the appropriate probabilities are computed from the results obtained in Section V as discussed in (21)-(24).

\section{EXPLICIT EXPRESSIONS FOR THE SYMMETRICAL MODEL FOR THE TWO-USER CASE}

In this section we consider the symmetrical model and we obtain closed form expressions for the average delay for the collision model and the capture model without explicitly computing the generating function for the stationary joint queue length distribution. Moreover, we provide upper and lower delay bounds for the MPR channel model.

By symmetrical, we mean the case where $\alpha_{k}^{*}=\alpha^{*}$, $\alpha_{k}=\alpha, \lambda_{k}=\lambda, P_{k /\{k\}}=p, \tilde{P}_{k /\{k\}}=\tilde{p}, P_{k /\{1,2\}}=b$, $P_{1,2 /\{1,2\}}=c, k=1,2$. Due to the symmetry of the model we have $H_{1}(1,1)=H_{2}(1,1), H_{1}(1,0)=H_{2}(0,1)$. Recall that $L_{k}=H_{k}(1,1)$ is the expected number of packets in node $k$. Therefore, after simple calculations using (6) we obtain,

$$
L_{1}=\frac{\lambda+\left(d+\alpha^{2} c\right) H_{1}(1,0)}{\alpha(p+\alpha(b+c-p))-\lambda} .
$$

Setting $x=y$ in (6), differentiating it with respect to $x$ at $x=1$, and using (7) we obtain

$$
\begin{aligned}
& L_{1}+L_{2}=2 L_{1}= \\
& \frac{2 \lambda-\lambda^{2}+\alpha^{2} c P\left(N_{1}>0, N_{2}>0\right)+2 H_{1}(1,0)\left(\alpha\left(p+\alpha(b-p)+d+2 \alpha^{2} c\right)\right)}{2(\alpha(p+\alpha(b+c-p))-\lambda)} .
\end{aligned}
$$

Using (25), (26) we finally obtain

$$
\begin{aligned}
L_{1}=L_{2}= & \frac{\lambda\left[2\left(\alpha+\alpha^{2}(b+c-p)\right)+\lambda\left(d+\alpha^{2} c\right)\right]}{2 \alpha^{*} \tilde{p}(\alpha(p+\alpha(b+c-p))-\lambda)} \\
& -\frac{\alpha^{2} c\left(d+\alpha^{2} c\right) P\left(N_{1}>0, N_{2}>0\right)}{2 \alpha^{*} \tilde{p}(\alpha(p+\alpha(b+c-p))-\lambda)} .
\end{aligned}
$$

Therefore, using Little's law the average delay in a node is given by

$$
D_{1}=D_{2}=\frac{2\left(\alpha+\alpha^{2}(b+c-p)\right)+\lambda\left(d+\alpha^{2} c\right)}{2 \alpha^{*} \tilde{p}(\alpha(p+\alpha(b+c-p))-\lambda)}+\phi,
$$

where $\phi=-\frac{\alpha^{2} c\left(d+\alpha^{2} c\right) P\left(N_{1}>0, N_{2}>0\right)}{2 \lambda \alpha^{*} \tilde{p}(\alpha(p+\alpha(b+c-p))-\lambda)}$; note that $\alpha(p+\alpha(b+$ $c-p))>\lambda$ due to the stability condition.

In case of the capture model, i.e., $c=0$, the exact average queueing delay in a node is given by (28) for $\phi=0$. In case $c \neq 0$, i.e., strong MPR effect, we are going to obtain upper and lower bounds for the expected delay based on the sign of $\phi$. Since $P\left(N_{1}>0, N_{2}>0\right)>0$, the sign of $\phi$ coincides with the sign of $d+\alpha^{2} c$. Thus, in order to proceed, we distinguish the analysis in the following two cases:

1) If $d+\alpha^{2} c<0$, then $0 \leq \phi \leq-\frac{\alpha^{2} c\left(d+\alpha^{2} c\right)}{2 \lambda \alpha^{*} \tilde{p}(\alpha(p+\alpha(b+c-p))-\lambda)}$. Thus, the upper and lower delay bound, say $D_{1}^{l o w}, D_{1}^{u p}$ respectively are,

$$
\begin{aligned}
D_{1}^{\text {low }} & =\frac{2\left(\alpha+\alpha^{2}(b+c-p)\right)+\lambda\left(d+\alpha^{2} c\right)}{2 \alpha^{*} \tilde{p}(\alpha(p+\alpha(b+c-p))-\lambda)}, \\
D_{1}^{\text {up }} & =D_{1}^{\text {low }}-\frac{\alpha^{2} c\left(d+\alpha^{2} c\right)}{2 \lambda \alpha^{*} \tilde{p}(\alpha(p+\alpha(b+c-p))-\lambda)} .
\end{aligned}
$$

2) If $d+\alpha^{2} c>0$, then $-\frac{\alpha^{2} c\left(d+\alpha^{2} c\right)}{2 \lambda \alpha^{*} \tilde{p}(\alpha(p+\alpha(b+c-p))-\lambda)} \leq \phi \leq 0$. In such a case,

$$
\begin{aligned}
D_{1}^{u p} & =\frac{2\left(\alpha+\alpha^{2}(b+c-p)\right)+\lambda\left(d+\alpha^{2} c\right)}{2 \alpha^{*} \tilde{p}(\alpha(p+\alpha(b+c-p))-\lambda)}, \\
D_{1}^{\text {low }} & =D_{1}^{u p}-\frac{\alpha^{2} c\left(d+\alpha^{2} c\right)}{2 \lambda \alpha^{*} \tilde{p}(\alpha(p+\alpha(b+c-p))-\lambda)} .
\end{aligned}
$$

Remark 4. Recall that $d+\alpha^{2} c$, is the difference of the successful transmission probability of a node when both nodes are active minus the successful transmission probability of a node when the other node is inactive; see Remark 1. Thus, it is realistic to assume that $d+\alpha^{2} c<0$, since it is more likely for a node to successfully transmit a packet when it is the only active. 
The next remark presents a closed form expression for the gap between the upper bound and the lower bound of the average delay.

Remark 5. We observe that in both cases described previously we have that

$$
D_{1}^{u p}-D_{1}^{\text {low }}=\left|\frac{\alpha^{2} c\left(d+\alpha^{2} c\right)}{2 \lambda \alpha^{*} \tilde{p}(\alpha(p+\alpha(b+c-p))-\lambda)}\right| .
$$

For the capture channel model, i.e., $c=0$, we have that $D_{1}^{u p}=D_{1}^{\text {low }}$ which is the exact delay. For the MPR model, we observe that as $\lambda$ increases then $D_{1}^{u p}-D_{1}^{\text {low }} \rightarrow 0$. In the very low arrival rate $\lambda$ regime, i.e., when $\lambda \rightarrow 0$, the upper bound of delay seems not to be tight. However, in such a case, well known results from queueing theory indicate that the delay is very close to zero, and thus, the lower bound on the delay is tight. In the next section we evaluate numerically the performance and we illustrate that the bounds are tight.

The next lemma provides the optimal transmission probability that minimizes the average delay for the symmetric capture channel model.

Lemma VII.1. Let $\tilde{\alpha}$ be the optimal transmission probability for the minimizing the expected delay in the symmetric capture channel model with $\alpha \leq \alpha^{*} \leq 1$. Then,

$$
\tilde{\alpha}= \begin{cases}\alpha^{*}, & \text { if } b \geq \frac{p\left(2 \alpha^{*}-1\right)}{2 \alpha^{*}}, \\ \frac{p}{2(p-b)}, & \text { if } 0 \leq b<\frac{p\left(2 \alpha^{*}-1\right)}{2 \alpha^{*}} .\end{cases}
$$

Proof. The problem can be cast as follows:

$$
\tilde{\alpha}=\underset{\left\{\alpha(p+\alpha(b-p))-\lambda>0, \alpha \in\left[0, \alpha^{*}\right]\right\}}{\operatorname{argmin}}\left\{\frac{(2+\lambda)\left(\alpha+\alpha^{2}(b-p)\right)+\lambda \alpha^{*} \tilde{p}}{2 \alpha^{*} \tilde{p}(\alpha(p+\alpha(b-p))-\lambda)}\right\} .
$$

To proceed, we first focus on the looser constrained optimization problem,

$$
\alpha^{\prime}=\underset{\{\alpha(p+\alpha(b-p))-\lambda>0\}}{\operatorname{argmin}}\left\{\frac{(2+\lambda)\left(\alpha+\alpha^{2}(b-p)\right)+\lambda \alpha^{*} \tilde{p}}{2 \alpha^{*} \tilde{p}(\alpha(p+\alpha(b-p))-\lambda)}\right\} .
$$

Clearly $b<p$, since it is more likely a transmission to be successful when only one node is transmitting rather than when both nodes transmit. Thus, $\alpha(p+\alpha(b-p))-\lambda>0$ is equivalent with $s_{1}<\alpha<s_{2}$, where $s_{1}, s_{2}$ the roots of $\alpha(p+\alpha(b-p))-\lambda=0$, where $0 \leq s_{1} \leq 1$. Differentiating the objective function in (30), we can easily derive that the only possible minimum is be given at $\alpha^{\prime}=\frac{p}{2(p-b)}$, where $s_{1} \leq \alpha^{\prime} \leq s_{2}$. If $\alpha^{\prime}<\alpha^{*}$, which is true for $b<\frac{p\left(2 \alpha^{*}-1\right)}{2 \alpha^{*}}$, then $\tilde{\alpha}=\alpha^{\prime}=\frac{p}{2(p-b)}$ is the minimum of the objective function (29). If $\alpha^{\prime} \geq \alpha^{*}$, which is equivalent with $b \geq \frac{p\left(2 \alpha^{*}-1\right)}{2 \alpha^{*}}$, then the optimal transmission probability, which minimizes the objective function in (29) is $\tilde{\alpha}=\alpha^{*}$.

\section{NUMERICAL RESULTS}

In this section, we provide numerical results to validate the analysis presented earlier. We consider the case where the users have the same link characteristics and transmission probabilities to facilitate exposition clarity, so we will use the notation from Section VII. In order to validate our theoretical results we built a MATLAB-based behavioral simulator. Not surprisingly, the simulation and theoretical results coincide after 15000 time slots.

\section{A. Stable Throughput Region}

The stability or stable throughput region for given transmission probabilities is depicted in Fig. 1 in the general case. The proposed random access scheme for given transmission probabilities is superior in the cases of collision, capture and the MPR channel modes, as it can be easily seen by replacing the parameters and putting $\alpha_{1}^{*}=\alpha_{1}$ and $\alpha_{2}^{*}=\alpha_{2}$.

As mentioned above, in Section III, we obtained the stability region with fixed transmission probability vectors $\left(\alpha_{1}, \alpha_{2}, \alpha_{1}^{*}, \alpha_{2}^{*}\right)$. If we take the union of these regions over all possible transmission probabilities of the users, we obtain the total stability region (i.e. the envelope of the individual regions). This corresponds to the closure of the stability region and is defined as

$$
\begin{aligned}
\mathcal{F} \triangleq & \left(\bigcup_{\overrightarrow{\boldsymbol{\alpha}} \in[0,1]^{2} \times\left[\alpha_{1}, 1\right] \times\left[\alpha_{2}, 1\right]} \mathcal{F}_{1}(\overrightarrow{\boldsymbol{\alpha}})\right) \\
& \bigcup\left(\bigcup_{\overrightarrow{\boldsymbol{\alpha}} \in[0,1]^{2} \times\left[\alpha_{1}, 1\right] \times\left[\alpha_{2}, 1\right]} \mathcal{F}_{2}(\overrightarrow{\boldsymbol{\alpha}})\right),
\end{aligned}
$$

where $\mathcal{F}_{i}(\overrightarrow{\boldsymbol{\alpha}}) \triangleq \mathcal{R}_{i}$ for $i=1,2$ are obtained in Section III and $\overrightarrow{\boldsymbol{\alpha}}=\left(\alpha_{1}, \alpha_{2}, \alpha_{1}^{*}, \alpha_{2}^{*}\right)$ is the vector of transmission probabilities.

Here, we will present the closure of the stable throughput region for the collision channel case where $p=\tilde{p}=1$ and $b=c=0 . .^{5}$ In Figs. 2(a) and 2(b) the closure of the stability region for the traditional collision channel with random access and for the proposed scheme are depicted. Clearly, our scheme is superior to the traditional one. The region in Fig. 2(b) is broader than the one in Fig. 2(a) which means that higher arrival rates can be supported and still maintain the system stable. Besides, the shape of the closure of the proposed scheme has linear behavior compare to the non-linear for the traditional one. This is a very interesting result.

In Figs. 3(a) and 3(b) the closure of the stability region for the capture channel with random access and for the proposed scheme are depicted for $b=0.2$. Our scheme is still superior to the traditional one since the region in Fig. 3(a) is a subset of the region in Fig. 3(a).

\section{B. Average Delay}

The effect of the arrival rate $\lambda$ at the average delay is depicted in Fig. 4(a) for the collision, capture and the MPR channel models. We consider the case with $\alpha=0.6, \alpha^{*}=1$ and $p=0.9, \tilde{p}=1$. For the MPR channel we consider the case $b=0.4, c=0.2$. For the capture channel we have $b=0.4$ and for the collision channel $b=0$. Recall that for both collision and capture channel models $c=0$. Clearly, regarding the MPR channel model, the lower and the upper bounds appear to be close. As also expected the average delay is lower for the MPR than the capture and the collision. Naturally, finite delay can be sustained for larger values of $\lambda$ for the MPR case.

In Fig. 4(b) we present the effect of $\alpha^{*}$ on the average delay as $\lambda$ varies. The cases of the collision, capture and the MPR channel models are presented. As $\alpha^{*}$ increases then the

\footnotetext{
${ }^{5}$ The closure for the MPR channel model is omitted due to space limitations.
} 


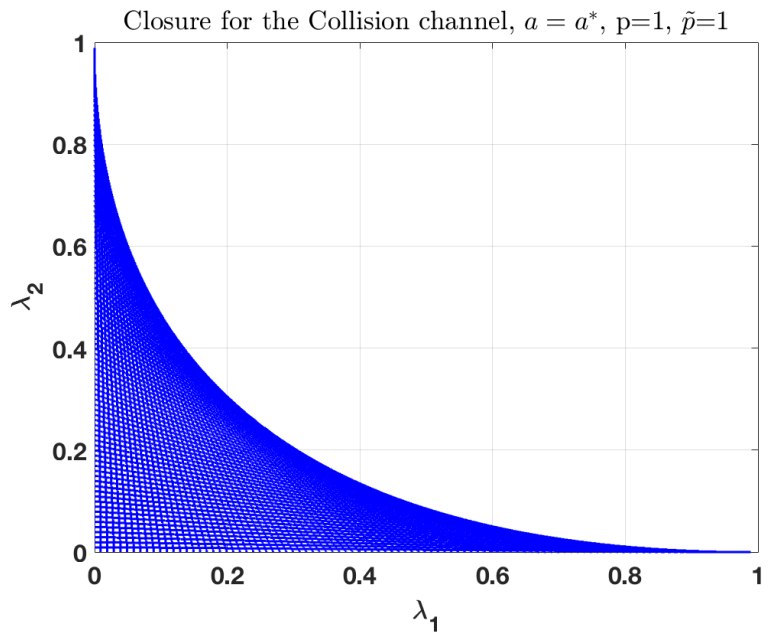

(a) Collision channel with $\alpha=\alpha^{*}$.

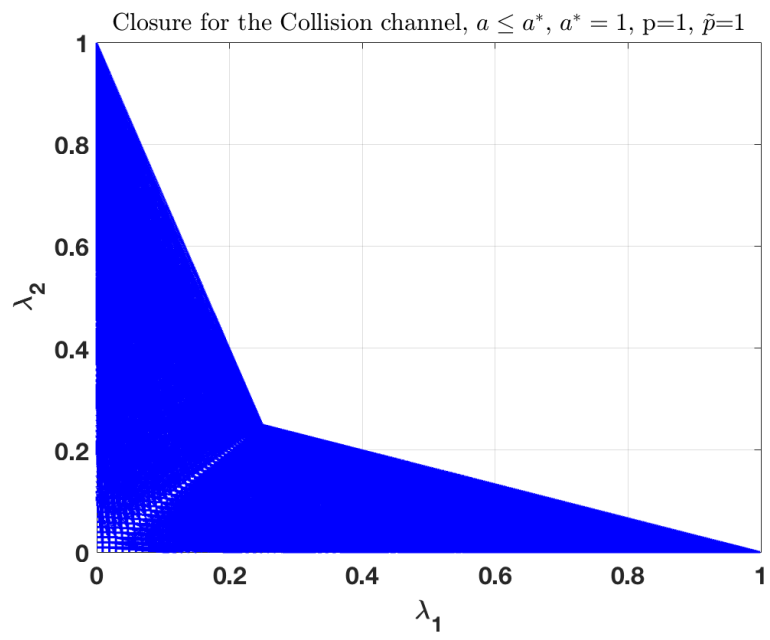

(b) Collision channel with $\alpha \leq \alpha^{*} \leq 1$.

Fig. 2. Closure of the Stability Region for the collision channel $(b=c=0)$ for $p=\tilde{p}=1$.

average delay decreases and also the maximum arrival rate that can still maintain a finite delay is getting larger. Adapting the transmission probabilities depending on the queue state can increase the performance of the system.

\section{CONCLUSION}

In this work we considered the case of the two and threeuser with bursty traffic in a random access wireless network with a common destination that has MPR capabilities. We assumed that the users adapt their transmission probabilities based on the status of the other nodes. For this network we provided the stability region for the two and the three-user case. For the two-user case we provided the convexity conditions of the stability region. Furthermore, we provided a detailed mathematical analysis and derived the generating function of the stationary joint queue length distribution of user nodes in terms of the solution of two boundary value problems. Based on that result we obtained expressions for the average queueing delay at each user node. For the two-user symmetric case with MPR we obtained a lower and an upper bound for the average delay without explicitly computing the generating function for

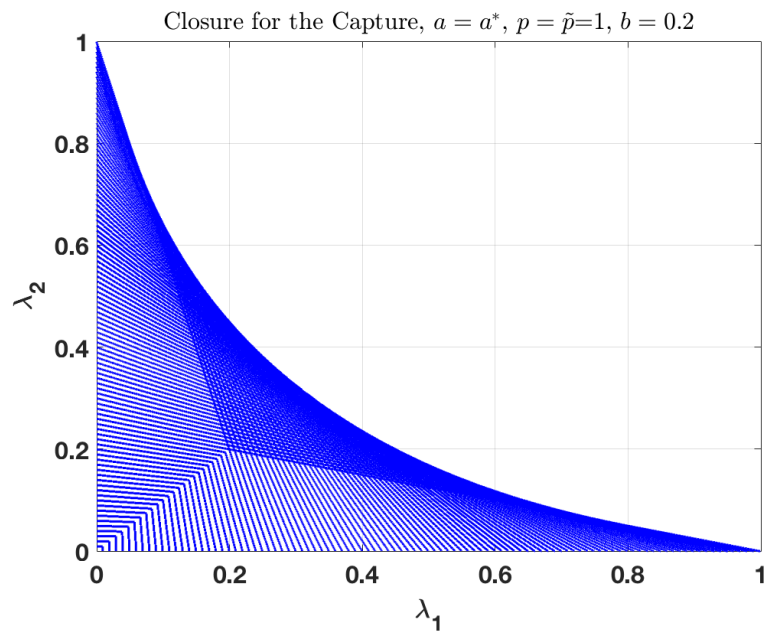

(a) Capture channel with $\alpha=\alpha^{*}$.

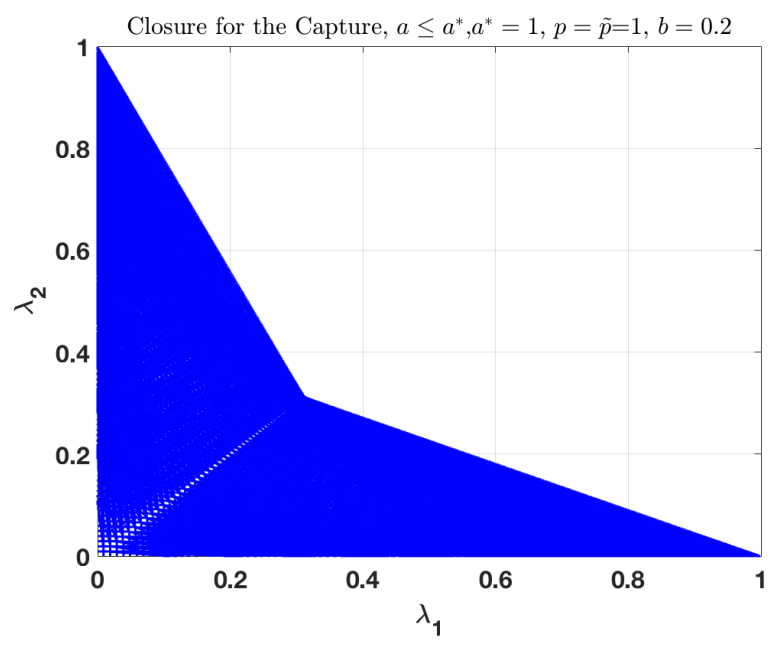

(b) Capture channel with $\alpha \leq \alpha^{*} \leq 1$.

Fig. 3. Closure of the Stability Region for the capture channel ( $b=0.2, c=$ $0)$ for $p=\tilde{p}=1$.

the stationary joint queue length distribution. The bounds as shown in the numerical results to be tight. Explicit expressions for the average delay are obtained for the model with capture effect. Finally, in the symmetric capture case, we obtained the optimal transmission probability in closed form expression that minimizes the average delay.

The analysis presented here can act as a framework for other research directions that involve interacting queues, such as the emerging area of Age of Information. Our next step is to use the current work as a building block in extending our results to the $N$-user scenario $(N>2)$, in which the system is described by an $N$-dimensional Markov chain. By applying the generating function approach, we come up with an intricate functional equation. However, the main problem is that in such a case, this functional equation contains $N$ unknown boundary functions, which in turn it is rather elaborated to obtain them explicitly. However, some important properties can be studied without the explicit derivation of these functions. In particular, using some algebraic arguments, and by appropriately differentiating the functional equation, we can obtain upper and lower bounds for the average queue 


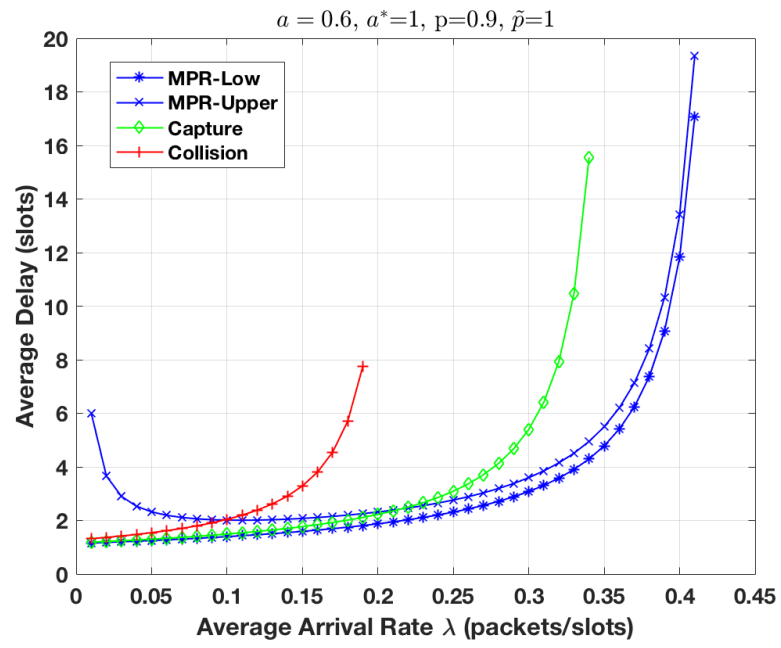

(a) Effect of $\lambda$ on the average delay.

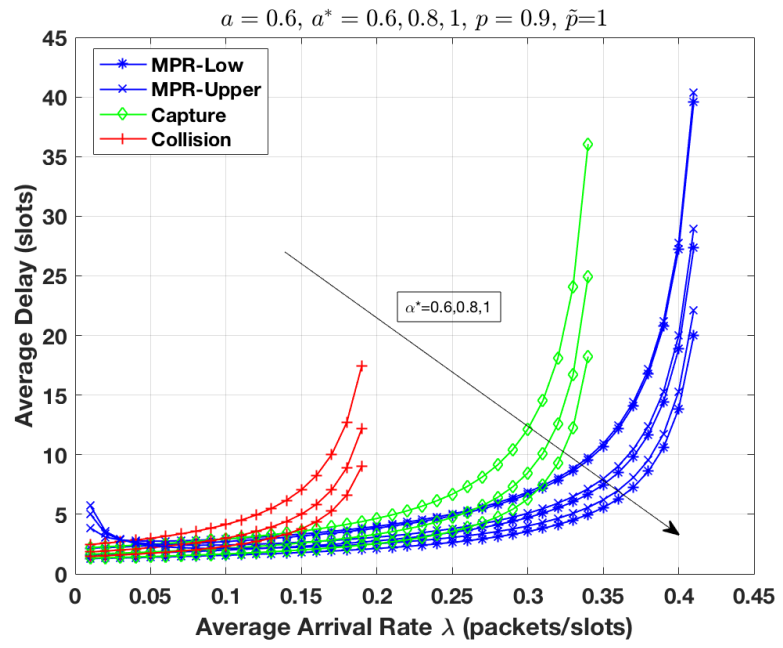

(b) Effect of $\alpha^{*}$ as $\lambda$ varies.

Fig. 4. Effect of $\lambda$ on the average delay for the collision, capture and the MPR channel models.

length in a buffer. Our preliminary results shows that the lower bound represents the average queue length in the $n$-th buffer, $n=1, \ldots, N$ under the condition that all the other buffers are always empty, which means that the bound is appropriate for a light traffic scenario. On the other hand, the upper bound is the average queue length in the $n$-th buffer under the condition that all the other buffers never empty, hence the bound is good for a heavy traffic scenario. For more sophisticated bounds, the general idea is to choose a set of users and determine the total average queue lengths of their buffers. Then, we can compare this average value with the sum of average queue lengths found by the appropriate differentiation of the functional equation in order to reduce the number of unknown boundary values. This final step will result in the derivation of the upper and the lower bounds.

\section{APPENDIX A}

\section{PROOF OF THEOREM III.1}

To determine the stability region of our system (depicted in Fig. 1) we apply the stochastic dominance technique [13], i.e. we construct hypothetical dominant systems, in which the source transmits dummy packets for the packet queue that is empty, while for the non-empty queue it transmits according to its traffic. Under this approach, we consider the $R_{1}$, and $R_{2}$ dominant systems. In the $R_{k}$ dominant system, whenever the queue of user $k, k=1,2$ empties, it continues transmitting a dummy packet. Thus, in $R_{1}$, node 1 never empties, and hence, node 2 sees a constant service rate, while the service rate of node 1 depends on the state of node 2 , i.e., empty or not. We proceed with queue at node 1 . The service rate of the first node is given by (2). The service rate of the second user is given by

$$
\mu_{2}=\alpha_{2} \bar{\alpha}_{1} P_{2 /\{2\}}+\alpha_{2} \alpha_{1}\left(P_{2 /\{1,2\}}+P_{1,2 /\{1,2\}}\right) .
$$

By applying Loyne's criterion, the second node is stable if and only if the average arrival rate is less that the average service rate, $\lambda_{2}<\alpha_{2} \bar{\alpha}_{1} P_{2 /\{2\}}+\alpha_{2} \alpha_{1}\left(P_{2 /\{1,2\}}+P_{1,2 /\{1,2\}}\right)$. We can obtain the probability that the second node is empty and is given by $\operatorname{Pr}\left(N_{2}=0\right)=1-\frac{\lambda_{2}}{\mu_{2}}$. After replacing $\operatorname{Pr}\left(N_{2}=0\right)$ into (2), and applying Loynes criterion we can obtain the stability condition for the first node. Then, we have the stability region $\mathcal{R}_{1}$ given by (3). Note that the expression in (3) is given in a more compact form that it will be useful in the next sections. Similarly, we can obtain the stability region for the second dominant system $\mathcal{R}_{2}$, the proof is omitted due to space limitations. For a detailed treatment of dominant systems please refer to [13].

An important observation made in [13] is that the stability conditions obtained by the stochastic dominance technique are not only sufficient but also necessary for the stability of the original system. The indistinguishability argument [13] applies here as well. Based on the construction of the dominant system, we can see that the queue sizes in the dominant system are always greater than those in the original system, provided they are both initialized to the same value and the arrivals are identical in both systems. Therefore, given $\lambda_{2}<\mu_{2}$, if for some $\lambda_{1}$, the queue at the first user is stable in the dominant system, then the corresponding queue in the original system must be stable. Conversely, if for some $\lambda_{1}$ in the dominant system, the queue at the first node saturates, then it will not transmit dummy packets, and as long as the first user has a packet to transmit, the behavior of the dominant system is identical to that of the original system since dummy packet transmissions are eliminated as we approach the stability boundary. Therefore, the original and the dominant system are indistinguishable at the boundary points.

\section{APPENDIX B ProOF OF LEMMA IV.1}

It is easily seen that $R(x, y)=\frac{x y-\Psi(x, y)}{x y D(x, y)}$, where $\Psi(x, y)=$ $D(x, y)\left[x y-y(x-1) \alpha_{1} \widehat{\alpha}_{2}-x(y-1) \alpha_{2} \widehat{\alpha}_{1}\right]$, where for $|x| \leq 1,|y| \leq 1, \Psi(x, y)$ is a generating function of a proper probability distribution. Now, for $|y|=1, y \neq 1$ and $|x|=1$ it is clear that $|\Psi(x, y)|<1=|x y|$. Thus, from Rouché's theorem, $x y-\Psi(x, y)$ has exactly one zero inside the unit circle. Therefore, $R(x, y)=0$ has exactly one root $x=X_{0}(y)$, such that $|x|<1$. For $y=1, R(x, 1)=0$ implies $(x-1)\left(\lambda_{1}-\frac{\alpha_{1} \widehat{\alpha}_{2}}{x}\right)=0$. Therefore, for $y=1$, and since $\lambda_{1}<\alpha_{1} \widehat{\alpha}_{2}$, the only root of $R(x, 1)=0$ for $|x| \leq 1$, is $x=1$. 


\section{APPENDIX C \\ PROOF OF LEMMA IV.3}

We will prove the part related to $\mathcal{M}$. Similarly, we can also prove part 2. For $y \in\left[y_{1}, y_{2}\right], D_{y}(y)$ is negative, so $X_{0}(y)$ and $X_{1}(y)$ are complex conjugates. Therefore, $|X(y)|^{2}=$ $\frac{\alpha_{1} \widehat{\alpha}_{2}}{\lambda_{1}\left(1+\lambda_{2}-\lambda_{2} y\right)}=g(y)$. Clearly, $g(y)$ is an increasing function for $y \in[0,1]$ and thus, $|X(y)|^{2} \leq g\left(y_{2}\right)$. Finally, $\zeta(\delta)$ is derived by solving $\operatorname{Re}(X(y))=-\widehat{b}(y) / 2 \widehat{a}(y)$ for $y$ with $\delta=\operatorname{Re}(X(y))$, and taking the solution such that $y \in[0,1] . \square$

\section{APPENDIX D}

\section{INTERSECTION POINTS OF THE CURVES}

In the following we focus on the location of the intersection points of $R(x, y)=0, A(x, y)=0$ (resp. $B(x, y)$ ). These points (if exist) are potential singularities for the functions $H(x, 0), H(0, y)$, and thus, their investigation is crucial regarding the analytic continuation of $H(x, 0), H(0, y)$ outside the unit disk; see also Lemma 2.2.1 and Theorem 3.2.3 in [40] for alternative approaches.

\section{A. Intersection points between $R(x, y)=0, A(x, y)=0$.}

Let $R(x, y)=0, x=X_{ \pm}(y), y \in \tilde{\tilde{C}}_{y}$. We can easily show that the resultant in $x$ of the two polynomials $R(x, y)$ and $A(x, y)$ is $\operatorname{Res}_{x}(R, A ; y)=y(y-1) Q(y)$, where $Q(y)=-\lambda_{2} d_{1}\left(d_{1}+\left(1+\lambda_{1}\right) \alpha_{2} \widehat{\alpha}_{1}\right) y^{2}+y \alpha_{2} \widehat{\alpha}_{1}\left[d_{1}(\lambda+\right.$ $\left.\left.\lambda_{1} \lambda_{2}\right)-\alpha_{1}^{*} \tilde{P}_{1 /\{1\}}\left(d_{1}+\alpha_{2} \widehat{\alpha}_{1}\right)\right]+\left(\alpha_{2} \widehat{\alpha}_{1}\right)^{2} \alpha_{1}^{*} \tilde{P}_{1 /\{1\}}$. Note that $Q(0)=\left(\alpha_{2} \widehat{\alpha}_{1}\right)^{2} \alpha_{1}^{*} \tilde{P}_{1 /\{1\}}>0$ and $Q(1)=d_{1}\left[\lambda_{1} \alpha_{2} \widehat{\alpha}_{1}-\right.$ $\left.\lambda_{2} d_{1}-\alpha_{2} \widehat{\alpha}_{1} \alpha_{1}^{*} \tilde{P}_{1 /\{1\}}\right]>0$, since $d_{1}<0$ and due to the stability condition.

Similarly, for $R(x, y)=0, y=Y_{ \pm}(x), x \in$ $\tilde{\mathrm{C}}_{x}$, the resultant in $y$ of the two polynomials $R(x, y)$, $A(x, y)$ is $\operatorname{Res}_{y}(R, A ; x)=x(x-1) \alpha_{2} \widehat{\alpha}_{1} \tilde{Q}(x)$, where $\tilde{Q}(x)=-\lambda_{1}\left(\alpha_{2} \widehat{\alpha}_{2}+\left(1+\lambda_{1}\right) d_{1}\right) x^{2}+x\left[\left(\lambda+\lambda_{1} \lambda_{2}\right) d_{1}+\right.$ $\left.\left(\alpha_{2} \widehat{\alpha}_{1}+d_{1}\right) \alpha_{1}^{*} \tilde{P}_{1 /\{1\}}\right]-\alpha_{1}^{*} \tilde{P}_{1 /\{1\}} d_{1}$. Note also that $\tilde{Q}(0)=$ $-\alpha_{1}^{*} \tilde{P}_{1 /\{1\}} d_{1}>0$ since $d_{1}<0$ and $\tilde{Q}(1)=\alpha_{2} \widehat{\alpha}_{1} \alpha_{1}^{*} \tilde{P}_{1 /\{1\}}-$ $\lambda_{1} \alpha_{2} \widehat{\alpha}_{1}+\lambda_{2} d_{1}>0$ due to the stability conditions (see Lemma III.1). If $\alpha_{1}^{*} \leq \min \left\{1, \frac{\alpha_{2} \widehat{\alpha}_{1}+\left(1+\lambda_{2}\right) \alpha_{1} \widehat{\alpha}_{2}}{\left(1+\lambda_{2}\right) \tilde{P}_{1 /\{1\}}}\right\}$, then $\lim _{x \rightarrow \infty} \tilde{Q}(x)=-\infty$, and $\tilde{Q}(x)=0$ has two roots of opposite sign, say $x_{*}<0<1<x^{*}$. If $\frac{\alpha_{2} \widehat{\alpha}_{1}+\left(1+\lambda_{2}\right) \alpha_{1} \widehat{\alpha}_{2}}{\left(1+\lambda_{2}\right) \tilde{P}_{1 /\{1\}}}<$ $\alpha_{1}^{*} \leq 1$, then $\lim _{x \rightarrow \infty} \tilde{Q}(x)=+\infty$, and $\tilde{Q}(x)=0$ has two positive roots, say $1<\tilde{x}_{*}<x_{3}<x_{4}<\tilde{x}^{*}$ (due to the stability conditions). In the former case we have to check if $x^{*}$ is in $S_{x}$, while in the latter case if $\tilde{x}_{*}$ is in $S_{x}$. These zeros, if they lie in $S_{x}$ such that $\left|Y_{0}(x)\right| \leq 1$, are poles of $H(x, y)$. Denote by

$$
\bar{x}= \begin{cases}x^{*}, & \alpha_{1}^{*} \leq \min \left\{1, \frac{\alpha_{2} \widehat{\alpha}_{1}+\left(1+\lambda_{2}\right) \alpha_{1} \widehat{\alpha}_{2}}{\left(1+\lambda_{2}\right) \tilde{P}_{1 /\{1\}}}\right\}, \\ \tilde{x}_{*}, & \frac{\alpha_{2} \widehat{\alpha}_{1}+\left(1+\lambda_{2}\right) \alpha_{1} \widehat{\alpha}_{2}}{\left(1+\lambda_{2}\right) \tilde{P}_{1 /\{1\}}}<\alpha_{1}^{*} \leq 1\end{cases}
$$

\section{$B$. Intersection points between $R(x, y)=0, B(x, y)=0$.}

Let $y \in \tilde{\tilde{\mathrm{C}}}_{y}$ and $R(x, y)=0, x=X_{ \pm}(y)$. It is easily shown that

$$
\begin{aligned}
R(x, y)= & B(x, y)+\lambda_{1}(1-x)+\lambda_{2}(1-y) \\
& +\lambda_{1} \hat{\lambda}_{2}(1-x)(1-y)+\alpha_{2}^{*} \tilde{P}_{2 /\{2\}}\left(1-\frac{1}{y}\right) .
\end{aligned}
$$

Thus, $R(x, y)=0, B(x, y)=0$ implies that,

$$
\begin{aligned}
\lambda_{1} x y(1-x)+\lambda_{2} x y(1-y) & \\
+\lambda_{1} \lambda_{2} x y(1-x)(1-y)+\alpha_{2}^{*} \tilde{P}_{2 /\{2\}} x(y-1) & =0, \\
\alpha_{1} \widehat{\alpha}_{2} y(x-1)+d_{2} x(y-1) & =0 .
\end{aligned}
$$

The second equation gives $x=\alpha_{1} \widehat{\alpha}_{2} y /\left(\alpha_{1} \widehat{\alpha}_{2} y+d_{2}(y-\right.$ 1)). Substituting back in the first one yields, $\tilde{W}(y)=$ $\frac{y-1}{y\left(\alpha_{1} \widehat{\alpha}_{2} y+d_{2}(y-1)\right)} \tilde{S}(y)$, where $\tilde{S}(y)=-\lambda_{2}\left(\alpha_{1} \widehat{\alpha}_{2}+d_{2}(1+\right.$ $\left.\left.\lambda_{1}\right)\right) y^{2}+y\left(d_{2}\left(\lambda+\lambda_{1} \lambda_{1}\right)+\alpha_{2}^{*} \tilde{P}_{2 /\{2\}}\left(d_{2}+\alpha_{1} \widehat{\alpha}_{2}\right)\right)-\alpha_{2}^{*} \tilde{P}_{2 /\{2\}} d_{2}$. Note that $\tilde{S}(0)=-\alpha_{2}^{*} \tilde{P}_{2 /\{2\}} d_{2}>0, \tilde{S}(1)=\alpha_{1} \widehat{\alpha}_{2} \alpha_{2}^{*} \tilde{P}_{2 /\{2\}}-$ $\widehat{\lambda}_{2} \alpha_{1} \widehat{\alpha}_{2}+\widehat{\lambda}_{1} d_{2}>0$, due to the stability conditions. If $\alpha_{2}^{*}<$ $\min \left\{1, \frac{\alpha_{1} \widehat{\alpha}_{2}+\left(1+\lambda_{1}\right) \alpha_{2} \widehat{\alpha}_{1}}{\left(1+\lambda_{1}\right) \tilde{P}_{2 /\{2\}}}\right\}, \lim _{y \rightarrow \infty} \tilde{S}(y)=-\infty$, and $\tilde{S}(y)$ has two roots of opposite sign, say $y_{*}, y^{*}$ such that $y_{*}<0<$ $1<y^{*}$, and $\tilde{W}(y)>0$ for $y \in(0,1)$, which in turn implies that $B\left(X_{0}(y), y\right) \neq 0, y \in\left[y_{1}, y_{2}\right] \subset(0,1)$, or equivalently $B\left(x, Y_{0}(x)\right) \neq 0, x \in \mathcal{M}$. When $\frac{\alpha_{1} \widehat{\alpha}_{2}+\left(1+\lambda_{1}\right) \alpha_{2} \widehat{\alpha}_{1}}{\left(1+\lambda_{1}\right) \tilde{P}_{2 /\{2\}}}<\alpha_{2}^{*} \leq 1$, $\lim _{y \rightarrow \infty} \tilde{S}(y)=+\infty$, and $\tilde{S}(y)$ has two positive roots, say $\widehat{y}_{*}, \widehat{y}^{*}$ such that $1<\widehat{y}_{*}<y_{3}<y_{4}<\widehat{y}^{*}$, and $\tilde{W}(y)>0$ for $y \in(0,1)$, which in turn implies that $B\left(X_{0}(y), y\right) \neq 0$, $y \in\left[y_{1}, y_{2}\right]$, i.e., $B\left(x, Y_{0}(x)\right) \neq 0, x \in \mathcal{M}$.

\section{REFERENCES}

[1] N. Abramson, "THE ALOHA SYSTEM: Another alternative for computer communications," in Proc., Fall Joint Computer Conference, ser. AFIPS. NY, USA: ACM, 1970, pp. 281-285. [Online]. Available: http://doi.acm.org/10.1145/1478462.1478502

[2] A. Laya, L. Alonso, and J. Alonso-Zarate, "Is the random access channel of LTE and LTE-A suitable for M2M communications? a survey of alternatives," IEEE Commun. Surveys Tutorials, vol. 16, no. 1, pp. 416, 2014.

[3] A. Osseiran, F. Boccardi, V. Braun, K. Kusume, P. Marsch, M. Maternia, O. Queseth, M. Schellmann, H. Schotten, H. Taoka, H. Tullberg, M. A. Uusitalo, B. Timus, and M. Fallgren, "Scenarios for 5G mobile and wireless communications: the vision of the METIS project," IEEE Commun. Mag., vol. 52, no. 5, pp. 26-35, May 2014.

[4] M. Koseoglu, "Lower bounds on the LTE-A average random access delay under massive M2M arrivals," IEEE Trans. on Commun., vol. 64, no. 5, pp. 2104-2115, May 2016.

[5] J. B. Seo and V. C. M. Leung, "Performance modeling and stability of semi-persistent scheduling with initial random access in lte," IEEE Trans. on Wir. Commun., vol. 11, no. 12, pp. 4446-4456, Dec. 2012.

[6] Z. Utkovski, O. Simeone, T. Dimitrova, and P. Popovski, "Random access in C-RAN for user activity detection with limited-capacity fronthaul," IEEE Sig. Proc. Letters, vol. 24, no. 1, pp. 17-21, Jan. 2017.

[7] H. Wang and T. Li, "Hybrid ALOHA: A novel MAC protocol," IEEE Trans. on Sig. Proc., vol. 55, no. 12, pp. 5821-5832, Dec. 2007.

[8] Z. Utkovski, T. Eftimov, and P. Popovski, "Random access protocols with collision resolution in a noncoherent setting," IEEE Wireless Commun. Letters, vol. 4, no. 4, pp. 445-448, Aug. 2015.

[9] C. Stefanovic and P. Popovski, "ALOHA random access that operates as a rateless code," IEEE Trans. on Commun., vol. 61, no. 11, pp. $4653-$ 4662, Nov. 2013.

[10] A. Ephremides and B. Hajek, "Information theory and communication networks: an unconsummated union," IEEE Trans. on Inf. Theory, vol. 44, no. 6, pp. 2416-2434, Oct. 1998.

[11] L. Tong, V. Naware, and P. Venkitasubramaniam, "Signal processing in random access," IEEE Sig. Proc. Mag., vol. 21, no. 5, pp. 29-39, Sep. 2004.

[12] Y. Gao, C. W. Tan, Y. Huang, Z. Zeng, and P. R. Kumar, "Characterization and optimization of delay guarantees for real-time multimedia traffic flows in IEEE 802.11 WLANs," IEEE Trans. on Mob. Comp., vol. 15, no. 5, pp. 1090-1104, May 2016.

[13] R. Rao and A. Ephremides, "On the stability of interacting queues in a multiple-access system," IEEE Trans. on Inf. Theory, vol. 34, no. 5, pp. 918-930, Sep. 1988.

[14] B. S. Tsybakov and V. A. Mikhailov, "Ergodicity of a slotted ALOHA system," Problemy Peredachi Informatsii, vol. 15, p. 7387, 1979. 
[15] W. Szpankowski, "Stability conditions for some distributed systems: buffered random access systems," Advances in Applied Probability, vol. 26, no. 2, pp. 498-515, 1994.

[16] W. Luo and A. Ephremides, "Stability of $\mathrm{N}$ interacting queues in random-access systems," IEEE Trans. on Inf. Theory, vol. 45, no. 5, pp. 1579-1587, July 1999.

[17] V. Naware, G. Mergen, and L. Tong, "Stability and delay of finite-user slotted ALOHA with multipacket reception," IEEE Trans. on Inf. Theory, vol. 51, no. 7, pp. 2636-2656, July 2005.

[18] C. Bordenave, D. McDonald, and A. Proutiere, "Asymptotic stability region of slotted ALOHA," IEEE Trans. on Inf. Theory, vol. 58, no. 9, pp. 5841-5855, Sept 2012.

[19] Y. Zhong, M. Haenggi, T. Q. S. Quek, and W. Zhang, "On the stability of static poisson networks under random access," IEEE Trans. on Commun., vol. 64, no. 7, pp. 2985-2998, July 2016.

[20] P. Nain, "Analysis of a two-node aloha-network with infinite capacity buffers," in Int. Seminar on Computer Networking and Performance Evaluation, Sep. 1985, pp. 49-63.

[21] A. B. Behroozi-Toosi and R. R. Rao, "Delay upper bounds for a finite user random-access system with bursty arrivals," IEEE Trans. on Commun., vol. 40, no. 3, pp. 591-596, Mar. 1992.

[22] L. Georgiadis, L. Merakos, and P. Papantoni-Kazakos, "A method for the delay analysis of random multiple-access algorithms whose delay process is regenerative," IEEE Journal on Sel. Areas in Commun., vol. 5, no. 6, pp. 1051-1062, Jul. 1987.

[23] K. Stamatiou and M. Haenggi, "Random-access poisson networks: Stability and delay," IEEE Commun. Letters, vol. 14, no. 11, pp. 10351037, Nov. 2010.

[24] S. Wang, J. Zhang, and L. Tong, "Delay analysis for cognitive radio networks with random access: A fluid queue view," in Proc., IEEE INFOCOM, Mar. 2010, pp. 1-9.

[25] N. Bouman, S. Borst, J. van Leeuwaarden, and A. Proutiere, "Backlogbased random access in wireless networks: Fluid limits and delay issues," in Proc., Intl. Teletraffic Congress (ITC), Sep. 2011, pp. 39-46.

[26] S. Srinivasa and M. Haenggi, "Throughput-delay-reliability tradeoffs in multihop networks with random access," in Proc., Allerton Conf. on Comm., Control, and Computing, Sep. 2010, pp. 1117-1124.

[27] _ - "A statistical mechanics-based framework to analyze ad hoc networks with random access," IEEE Trans. on Mob. Comp., vol. 11, no. 4, pp. 618-630, Apr. 2012.

[28] F. Poloczek and F. Ciucu, "Service-martingales: Theory and applications to the delay analysis of random access protocols," in Proc., IEEE INFOCOM, Apr. 2015, pp. 945-953.

[29] N. Pappas, M. Kountouris, A. Ephremides, and A. Traganitis, "Relayassisted multiple access with full-duplex multi-packet reception," IEEE Trans. on Wir. Commun., vol. 14, no. 7, pp. 3544-3558, July 2015.

[30] X. Jian, Y. Liu, Y. Wei, X. Zeng, and X. Tan, "Random access delay distribution of multichannel slotted ALOHA with its applications for machine type communications," IEEE Internet of Things Journal, vol. 4, no. 1, pp. 21-28, Feb. 2017.

[31] Z. Chen, N. Pappas, M. Kountouris, and V. Angelakis, "Throughput analysis of smart objects with delay constraints," in Proc. IEEE Intl. Symp. on A World of Wireless, Mobile and Multimedia Networks (WoWMoM), June 2016, pp. 1-6.

[32] K. Stamatiou and M. Haenggi, "Delay characterization of multihop transmission in a poisson field of interference," IEEE/ACM Trans. on Networking, vol. 22, no. 6, pp. 1794-1807, Dec. 2014.

[33] Q. Yao, A. Huang, H. Shan, T. Q. S. Quek, and W. Wang, "Delayaware wireless powered communication networks energy balancing and optimization," IEEE Trans. on Wir. Commun., vol. 15, no. 8, pp. 5272 5286, Aug. 2016

[34] E. Sandgren, A. G. i Amat, and F. Brännström, "On frame asynchronous coded slotted ALOHA: Asymptotic, finite length, and delay analysis," IEEE Trans. on Commun., vol. 65, no. 2, pp. 691-704, Feb. 2017.

[35] W. Szpankowski, "Stability conditions for multidimensional queueing systems with computer applications," Operations Research, vol. 36, no. 6, pp. 944-957, 1988. [Online]. Available: http://www.jstor.org/ stable/171125

[36] J. Cohen and O. Boxma, Boundary value problems in queueing systems analysis. Amsterdam, Netherlands: North Holland Publishing Company, 1983.

[37] I. Dimitriou, "A two class retrial system with coupled orbit queues," Prob. Engin. Infor. Sc., vol. 31, no. 2, pp. 139-179, 2017.

[38] _ "A queueing model with two types of retrial customers and paired services," Ann. Oper. Res., vol. 238, no. 1, pp. 123-143, 2016.
[39] G. Fayolle, R. Iasnogorodski, and V. Malyshev, Random walks in the quarter-plane: Algebraic methods, boundary value problems and applications, vol. 40 of Applications of Mathematics. Springer-Verlag, Berlin, 1999.

[40] G. Fayolle and R. Iasnogorodski, "Two coupled processors: The reduction to a riemann-hilbert problem," Zeitschrift für Wahrscheinlichkeitstheorie und Verwandte Gebiete, vol. 47, no. 3, pp. 325-351, 1979. [Online]. Available: http://dx.doi.org/10.1007/BF00535168

[41] K. Avrachenkov, P. Nain, and U. Yechiali, "A retrial system with two input streams and two orbit queues," Queueing Systems, vol. 77, no. 1, pp. 1-31, 2014. [Online]. Available: http://dx.doi.org/10.1007/ s11134-013-9372-8

[42] D. Tse and P. Viswanath, Fundamentals of wireless communication. NY, USA: Cambridge University Press, 2005.

[43] C. T. Lau and C. Leung, "Capture models for mobile packet radio networks," IEEE Trans. on Commun., vol. 40, no. 5, pp. 917-925, May 1992.

[44] R. Loynes, "The stability of a queue with non-independent inter-arrival and service times," Proc. Camb. Philos. Soc, vol. 58, no. 3, pp. 497-520, 1962.

[45] C. T. Lau and C. Leung, "Capture models for mobile packet radio networks," IEEE Trans. on Commun., vol. 40, no. 5, pp. 917-925, May 1992.

[46] M. Zorzi and R. R. Rao, "Capture and retransmission control in mobile radio," IEEE Journal on Sel. Areas in Commun., vol. 12, no. 8, pp. 1289-1298, Oct. 1994.

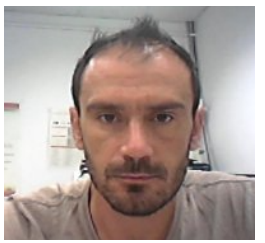

Ioannis Dimitriou (Ph.D. University of Ioannina, Greece, 2009) is a Lecturer in the Department of Mathematics, University of Patras, Greece. During October 2011 - July 2012, he was a postdoctoral researcher in the Department of Electrical and Electronic Engineering, Imperial College, London, UK. During January 2013-December 2013, he was a postdoctoral fellow in the Department of Mathematics, Aristotle University of Thessaloniki, Greece, while, during September 2013 - July 2014, he was a postdoctoral researcher in the ICS-FORTH, (Mobile Computing Group) Crete, Greece. For the time period, May 2014-July 2014 he was a postdoctoral fellow at INRIA, Sophia Antipolis (MAESTRO group) through the ERCIM Alain Bensoussan" Fellowship program (Marie Curie Actions).

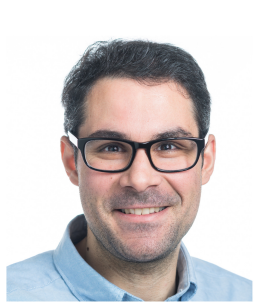

Nikolaos Pappas (S'07-M'13) received the B.Sc. degree in computer science, the B.Sc. degree in mathematics, the M.Sc. degree in computer science, and the Ph.D. degree in computer science from the University of Crete, Greece, in 2005, 2012, 2007 , and 2012, respectively. He is currently an Associate Professor of mobile telecommunications with the Department of Science and Technology, Linköping University, Norrköping, Sweden. From 2005 to 2012, he was a graduate Research Assistant with the Telecommunications and Networks Laboratory, Institute of Computer Science, Foundation for Research and Technology, Hellas, and a Visiting Scholar with the Institute of Systems Research, University of Maryland at College Park. From 2012 to 2014, he was a Post-Doctoral Researcher with the Department of Telecommunications, Supélec, France. Since 2014, he has been with the University of Linköping, Norrköping, as a Marie Curie Fellow (IAPP). His main research interests are in the field of wireless communication networks with emphasis on the stability analysis, energy harvesting networks, network-level cooperation, ageof-information, network coding, and stochastic geometry. From 2013 to 2018 he was Editor of the IEEE Communications Letters. 